\title{
Die peruanische Verfassung von 1993
}

\author{
Von Jürgen Saligmann
}

\section{Einleitung}

Mit der Volksabstimmung vom 31.10.1993 ist die 12. peruanische Verfassung seit der Staatsgründung im Jahre 1821 angenommen worden. Auch wenn die Zustimmung wohl als relativ zurückhaltend bezeichnet werden darf $^{1}$ - bei einer nicht unerheblichen Stimmenthaltung $^{2}$-, so soll an dieser Stelle nicht die grundsätzliche Frage nach der Legitimation der Verfassung aufgeworfen werden. Vielmehr wird zu untersuchen sein, ob diese Neufassung überhaupt notwendig war, ob hier wegen grundlegend veränderter Umstände in Politik und Gesellschaft eine neue Verfassung geschrieben werden mußte ${ }^{3}$ oder ob nicht doch eine punktuelle Reform ausreichend gewesen wäre ${ }^{4}$. Obwohl dem bis zum 5. April 1992 amtierenden Kongreß fast 100 Vorschläge zur Verfassungsänderung vorlagen, war wohl das Hauptproblem nicht die teilweise Revision, sondern vielmehr die Umsetzung der - bereits bestehenden - Grundsätze der Verfassung in die politische und soziale Realität ${ }^{5}$.

Zur Vorgeschichte: Alberto Fujimori hatte 1990, für die meisten in- und ausländischen Beobachter überraschend, die Präsidentschaftswahl gegen den vermeintlich haushohen Favoriten, den Schriftsteller Mario Vargas Llosa, gewonnen. Für dieses Phänomen hat es bereits zahlreiche politologische Erklärungsversuche gegeben ${ }^{6}$, die an dieser Stelle nicht

1

2

3

4

5

6

U.a. Fernando Rospigliosi, Las elecciones peruanas de 1990, in: R. Cerdas, J. Rial, D. Zovatto, Una tarea inconclusa: elecciones y democracia en América Latina, 1988-1991, San José , C.R., 1992, S. 345 ff.; Marcial Rubio, Gobernabilidad Democrática en el Perú, in: Análisis Internacional 3 (JuliSeptember) Lima 1993, S. 66 ff., der speziell auf die Politik- und Parteienverdrossenheit bei den Wählern hinweist. 
diskutiert werden sollen. Festzuhalten ist aber, daß der neue Präsident, im Gegensatz zu seinen Vorgängern, gegen eine starke Mehrheit im Kongreß regieren mußte. Am 5. April 1992 kam es dann zu dem sog. "Fujigolpe", einem Staatsstreich von oben. Der Präsident löste u.a. das Parlament auf und übernahm als Diktator die alleinige Regierungsgewalt.

Erheblicher politischer und ökonomischer Druck im In- und Ausland (so hatte die Bundesregierung umgehend die Entwicklungshilfe eingefroren) führten dann dazu, daß Fujimori am 22.11.1992 eine Verfassunggebende Versammlung wählen ließ. Nicht alle Parteien stellten dafür Kandidaten auf. So verweigerten u.a. die Acción Popular des früheren Präsidenten Fernando Belaúnde Terry (1980-85) sowie die APRA des Fujimori-Vorgängers Alan García Pérez (1985-90) ihre Beteiligung. Dafür werden hauptsächlich drei Gründe genannt: zum einen sollte der Putsch nicht nachträglich durch diesen Wahlakt legitimiert werden ${ }^{7}$. Des weiteren gab es wohl die Hoffnung auf einen Staatsstreich seitens des Militärs zum Schutz der (alten) Verfassung sowie des Rechtsstaats ${ }^{8}$. Und letztlich hatten diese etablierten Parteien aber auch Furcht vor einem für sie ungünstigen Wahlausgang ${ }^{9}$. Es tauchten neue Parteien und Bewegungen auf, die den verfassungsrechtlichen Vorstellungen des Präsidenten grundsätzlich ihre Unterstützung zugesagt hatten. In den folgenden knapp 12 Monaten wurde dann ein Verfassungsentwurf ausgearbeitet, der am 31.10.1993 mit knapper, aber ausreichender Mehrheit ${ }^{10}$ in einem Referendum angenommen wurde ${ }^{11}$. Die Verkündung erfolgte am 29.12.1993.

Die neue Verfassung enthält 206 Artikel, unterteilt in 6 Titel, die wiederum in verschiedene Kapitel aufgegliedert werden. Dazu kommen noch Übergangs- und Schlußbestimmungen. Der 1. Titel umfaßt grundlegende Vorschriften zu Person und Gesellschaft. Der 2. Titel handelt von Staat und Nation. Im 3. Titel geht es um das Wirtschaftssystem. Der 4. und mit über 100 Artikeln umfangreichste Titel beschreibt das Verhältnis der Staatsorgane zueinander. Titel 5 enthält verfassungsrechtliche Garantien, während der 6. Titel die Möglichkeiten einer Verfassungsreform normiert. Diese Gliederung entspricht im übrigen fast wörtlich der Verfassung von 1979. Die folgenden Ausführungen orientieren sich bewußt an dieser Aufteilung, da sie auch ein Exempel für das innewohnende Verfassungsverständnis darstellt.

Aus diesem Grund hatte im übrigen die Acción Popular schon die Verfassunggebende Versammlung von 1979 boykottiert, da F. Belaúnde T. 1968 durch einen Militärputsch als Präsident gestürzt worden war. Belaúnde ist dann aber aufgrund der Verfassung von 1979 im Mai 1980 wiederholt zum Staatsoberhaupt gewählt worden.

Federico Velarde, Las elecciones en el Perú. Lo que se dice y lo que no se dice, in: Socialismo y participación No. 65 (März 1994), S. 96.

Velarde, a.a.O.

10

S.o. Fußnote 1.

11

Die Verfassung von 1979 wurde nicht durch eine Volksabstimmung angenommen; vielmehr erfolgte die Schlußabstimmung durch die Verfassunggebende Versammlung. 


\section{Hauptteil}

\subsection{Präambel}

Die Präambel der neue Verfassung ist an Kürze und Inhaltsleere kaum zu überbieten: "Unter Berufung auf den allmächtigen Gott, dem Mandat des peruanischen Volkes gehorchend und in Erinnerung an das Opfer aller vorhergehenden Generationen in unserem Vaterland, hat die Demokratische Verfassunggebende Versammlung die folgende Verfassung beschlossen" (Übers. d. Verf.). Daraus kann auch nicht ansatzweise abgeleitet werden, welches die tragenden Elemente der Verfassung sein sollen ${ }^{12}$. Demgegenüber war die Verfassung von 1979 "durch eine sehr festliche, inhaltsreiche, die nachstehende Verfassung vorwegnehmende, barocke Präambel ${ }^{13}$ " aufgefallen ${ }^{14}$.

\subsection{Person und Gesellschaft}

Nach Art. 1 gehört es zu dem obersten Ziel von Gesellschaft und Staat, die Menschenwürde zu respektieren und zu schützen. Die persönlichen Rechte werden dann in Art. 2 durch insgesamt 24 Unterpunkte definiert, wobei der letzte Unterpunkt dann nochmals in 8 einzelne Kriterien aufgeteilt wird. Im Vergleich zu den im Grundgesetz sehr knapp gehaltenen Bestimmungen werden hier in sehr breiter Form die einzelnen Menschen- und Bürgerrechte ausgeführt.

Es soll hier nicht auf jede Bestimmung im einzelnen eingegangen werden, zumal sich die Aufzählung im allgemeinen an den in demokratischen Staaten üblichen Grundrechten orientiert. So gehört beispielsweise die allgemeine Religionsfreiheit dazu (Art. 2 Nr. 3), nicht ohne jedoch später, in Art. 50, die katholische Kirche als besonderes Element im historischen, kulturellen und moralischen Kontext der peruanischen Geschichte zu benennen und ihr die Zusammenarbeit anzubieten.

Erwähnenswert ist das Recht auf informationelle Selbstbestimmung ("hábeas data") ${ }^{15}$. Nach Art. 2 Nr. 5 kann jedermann gegenüber den Behörden einen Anspruch auf Informationsweitergabe geltend machen. Ausnahmen davon bilden Daten über die Intimsphäre, die nationale Sicherheit sowie dann, wenn ein Gesetz etwas anderes bestimmt. Dies ist

12

13

14

15

Zu dieser Rechtsfigur in lateinamerikanischem Verfassungsrecht s. Néstor Pedro Sagüés: Hábeas data: su desarrollo constitucional, in: Comisión Andina de Juristas: Lecturas Constitucionales Andinas No. 3, Lima 1994, S. 87 ff. 
tatsächlich, zumindest in der Theorie, eine gewichtige Änderung gegenüber der alten Verfassung. Art. 2 Nr. 6 untersagt es öffentlichen oder privaten Informationsdiensten, Daten zu speichern, die die persönliche und familiäre Privatsphäre beeinträchtigen könnten.

Umstritten ist jedoch die neue Regelung in Art. 2 Nr. 7: Bei einer persönlichen Ehrverletzung durch ein Medium gibt es nunmehr das Recht auf Gegendarstellung. Systematisch passe diese Bestimmung nicht zu dem allgemeinen Datenschutzrecht. Befürchtet wird, $\mathrm{da} ß$ damit indirekt eine Pressezensur ausgeübt werden kann ${ }^{16}$. In den ersten sechs Monaten nach Inkrafttreten dieser Verfassung waren bereits zwei Fälle unter Berufung auf den "hábeas data" gegen Presseorgane vor Gericht gekommen, allerdings in beiden Fällen ohne Erfolg ${ }^{17}$.

Des weiteren besteht nach Art. 2 Nr. 19 ein Recht auf ethnische und kulturelle Identität; der Staat anerkennt und schützt diese Pluralität. Im übrigen hat jeder Peruaner (und auch Ausländer) das Recht, sich seiner Muttersprache zu bedienen ${ }^{18}$; gegebenenfalls muß ihm ein Dolmetscher gestellt werden. Diese Bestimmung erscheint umso wichtiger, wenn man sich die seit Jahrhunderten übliche Diskriminierung gerade der indianischen Bevölkerung vor Augen hält, die in Peru immer noch, trotz aller gegenteiligen Beteuerungen amtlicherseits, an der Tagesordnung ist ${ }^{19}$.

$\mathrm{Da}$ im übrigen die Sklaverei (Art. 2 Nr. 24b) sowie der anachronistische Schuldturm (Art. 2 Nr. 24c) verboten werden, dokumentiert allerdings noch für die jüngere Vergangenheit gesellschaftliche Zustände, die, obwohl schon die Verfassung von 1979 derartige Regelungen enthielt, ein verfassungsrechtliches Verbot weiterhin notwendig machten.

Es darf allerdings nicht übersehen werden, daß auch in Peru zwischen Verfassungsrecht und Verfassungswirklichkeit ein Dissens besteht. So erklärt Art. 2 Nr. 24e jedermann für unschuldig, solange seine Verantwortlichkeit nicht gerichtlich festgestellt wurde. In krassem Gegensatz dazu steht die vom Verfasser beobachtete Praxis, all die, die des Terrorismus auch nur verdächtigt werden, unmittelbar nach ihrer Festnahme in schwarz-weiß gestreifte Kleider zu stecken und der Öffentlichkeit, d.h. insbesondere den Fernsehkame-

Samuel Abad Yupanqui: Hábeas Data y conflicto entre órganos constitucionales, in: Comisión Andina de Juristas, La Constitución de 1993, Lima 1994, S. 268 ff.

Nach Marcial Rubio Correa: El sistema legislativo en la Constitución de 1993, in: Comisión Andina de Juristas, La Constitución de 1993, Lima 1994, S. 167.

18

Nach Art. 48 sind neben Spanisch auch Ketschua, Aimara und die Sprachen der Naturvölker (speziell in der Amazonasregion) offizielle Sprachen, wenn es gesetzlich vorgesehen ist.

19

Francisco Fernández Segado: El nuevo ordenamiento constitucional del Perú, in: Comisión Andina de Juristas: La Constitución de 1993, Lima 1994, S.17 f. 
ras, vorzuführen. Erst Anfang 1995 ist festgelegt worden, daß diese Praxis zukünftig nur noch bei einigen wenigen, als besonders gefährlich geltenden Personen nach ihrer Festnahme beibehalten werden dürfe. Die Aufzählung der Rechte in Art. 2 ist nicht abschlieBend; vielmehr lassen sich u.a. aus den allgemeinen Menschenrechten weitere Grundrechte ableiten, Art. 3.

Zieht man zum Vergleich die Behandlung der Grundrechte in der Verfassung von 1979 heran, so wird man einige Differenzen feststellen können, deren Bedeutung jedoch eher marginaler Natur seien dürfte. Die - mit dem Inkrafttreten der Verfassung von 1979 - im Jahre 1980 einsetzende Redemokratisierung Perus nach 12-jähriger Militärherrschaft wurde begleitet von dem im gleichen Jahr einsetzenden Terror des "Sendero Luminoso" (Leuchtender Pfad). Der Versuch einer ausschließlich militärischen Bekämpfung dieser Subversion führte jedoch zu schwerwiegenden Beschränkungen von Grundrechten, speziell dem Recht auf persönliche Freiheit ${ }^{20}$.

Hinzu kommt noch die Frage nach dem Stellenwert der Grundrechte in der alten Verfassung im Verlauf ihrer 12-jährigen Existenz. Unstreitig sind die alten Verfassungsgrundsätze überwiegend Theorie geblieben; die Verfassungswirklichkeit war weit davon entfernt. Dennoch, oder gerade deshalb, wird gefordert, die Theorie als Richtschnur für das rechtliche und politische Handeln zu verwenden ${ }^{21}$. So normierte Art. 2 Nr. 2 der Verfassung von $1979^{22}$ i.V.m. Art. 43 ('79) das Gleichbehandlungsgebot in den arbeitsrechtlichen Beziehungen ausdrücklich dahingehend, Männern und Frauen bei gleicher Arbeit unter identischen Bedingungen einen Anspruch auf gleiche Bezahlung zu geben. Dies wurde gemeinhin als Selbstverständlichkeit aufgefaßt, was keiner weiteren Kommentierung mehr bedürfe ${ }^{23}$. Wohl verweisen Art. 23 und 24 auch auf den Grundsatz der Gleichbehandlung, ohne jedoch hier ausdrücklich auf diejenige von Männern und Frauen einzugehen. Kritisch wird dazu angemerkt, wohl werde dem formalen Gleichheitsgebot Genüge getan; die realen Lebensumstände würden so aber vergessen ${ }^{24}$.

Die Todesstrafe ist, in Anlehnung an Art. 235 ('79), nach Art. 140 bei Landesverrat im Kriegsfall sowie, und das ist neu, jetzt auch gegenüber Terroristen anwendbar. Über den

Francisco Eguiguren P.: Los retos de una democracia insuficiente, Lima 1990, S. 76 [zit: F. Eguiguren, Los retos...].

CAJ, Del golpe..., S. 206 f.

22

Im folgenden abgekürzt: ('79).

23

Marcial Rubio /Enrique Bernales: Constitución y sociedad política, 3. Aufl. Lima 1988, S. 598 [zit: Rubio/Bernales, Constitución]. rung von Art. 3 Abs. 2 GG (BT-Drucksache 12/6000 vom 05.11.93, S. 49 ff.)]. 
Sinn der Todesstrafe soll an dieser Stelle nicht diskutiert werden ${ }^{25}$. Diese Neuerung war im Vorfeld der Verfassungsdebatte höchst umstritten; jedoch hatte sich der Präsident mit seinem eher populistisch zu nennenden Entwurf durchsetzen können.

Besonders betont wird im politischen Lager von Präsident Fujimori, daß in Art. 2 Nr. 17 das Recht auf Partizipation der Bürger am politischen, ökonomischen, sozialen und kulturellen Leben nicht nur, wie in Art. 2 Nr. 16 ('79), pauschal eingeräumt wird, sondern ausdrücklich dabei noch das Recht auf Wahl und Abwahl von staatlichen Autoritäten sowie das Gesetzesinitiativrecht und das Referendum als Bürgerrechte normiert werden $^{26}$. Erst bei der Umsetzung in den entsprechenden Ausführungsgesetzen wird die wahre Substanz dieser Neuerung erkennbar werden ${ }^{27}$.

Wie in anderen Staaten Lateinamerikas üblich gilt auch in Peru laut Art. 31 die allgemeine Wahlpflicht, und zwar vom 18. bis zum 70. Lebensjahr. Ältere Bürger sind von der Pflicht befreit, haben aber natürlich weiterhin das Wahlrecht. Dies entspricht Art. 65 ('79). Beibehalten in dieser neuen Verfassung wurde die Regelung, daß die im aktiven Dienst stehenden Angehörigen von Polizei und Streitkräften weder das aktive noch das passive Wahlrecht haben, Art. 34 bzw. Art. 67 ('79). Das ist nicht unumstritten. Für die Beibehaltung dieser althergebrachten Regelung wird angeführt, nur so könne verhindert werden, daß dem an Befehl und Gehorsam gewöhnten einfachen Soldaten die Wahlentscheidung durch seine Vorgesetzten aufgezwungen werde. Im übrigen sei die Geschichte Perus geprägt durch eine konstante Einmischung der Militärs in das politische Leben, auch wenn das in den Verfassungen nie so vorgesehen gewesen sei. Diese Interventionen solle man nicht auch noch durch die Gewährung des Wahlrechts quasi legitimieren ${ }^{28}$. Die Gegenposition verweist darauf, daß gerade aufgrund der permanenten Einmischung des Militärs in die Politik während der vergangenen 170 Jahre die Frage des Wahlrechts völlig unerheblich sei. Zudem wäre es etwas simpel, schon aus dem Über- und Unterordnungsverhältnis innerhalb der Streitkräfte abzuleiten, ein Soldat würde in der Wahlkabine sein Kreuz bef ehlsgemäß an einer bestimmten Stelle machen; ähnliches könnte man dann auch dem Arbeiter unterstellen, der sich seine Wahlentscheidung von dem Unternehmer vorschreiben ließe $\mathrm{e}^{29}$.

Zur Diskussion in Peru siehe CAJ, Del golpe..., S. $208 \mathrm{ff}$.

Carlos Torres y Torres Lara, Alcances de la nueva Constitución del Perú de 1993, in: Análisis Internacional 4 (Oktober-Dezember) Lima, 1993, S. 4 ff.

CAJ, Del golpe..., S. 214 f.

José Pareja Paz Soldán, Derecho Constitucional Peruano y la Constitución de 1979, Lima 1980, 2. Band, S. 797 f.

29

Rubio/Bernales, Constitución, S. 655 ff. 
Im übrigen wäre auch zu überlegen, ob nicht durch das Wahlrecht die Streitkräfte besser in das politische Leben integriert werden können; zudem würde damit eventuell auch die Mitverantwortung der Militärs in einem demokratischen System gestärkt. Interessanterweise ist es im allgemeinen die politische Linke, die einem Wahlrecht für Soldaten aufgeschlossen gegenübersteht, während auf der Rechten um die Disziplin in den Kasernen gefürchtet wird, wenn erst der Wahlkampf in die Kasernen hineingetragen würde ${ }^{30}$.

Umstritten ist auch noch, wie der Sektor "Erziehung" in der neuen Verfassung behandelt wird. Nach Art. 25 ('79) bestand nur eine Schulpflicht für die Primarschule, d.h. die ersten fünf Schuljahre. Art. 17 hat nunmehr auch die nächsten fünf Jahre in der Sekundarschule für obligatorisch erklärt. Immer war und ist der Besuch nationaler Schulen aber kostenlos. Das ist insoweit unumstritten; problematischer (aber nicht aus juristischer Sicht) ist allenfalls, daß besonders außerhalb der Städte, in den abgelegenen Regionen, die Eltern ihre Kinder immer noch eher auf den Acker als zur Schule schicken.

Eine viel diskutierte Änderung betrifft den Besuch einer öffentlichen Hochschule. Auch der soll, so Art. 17, kostenlos sein, allerdings mit der Einschränkung, daß der Student seine Bedürftigkeit und eine genügende Leistung nachweisen mu $\beta^{31}$. Ihre Bedürftigkeit werden die Betroffenen nachweisen können; allerdings wird der geforderte Leistungsnachweis schwieriger zu erbringen sein, weil viele Studenten an den öffentlichen Universitäten, da bedürftig, nebenbei arbeiten und Geld verdienen müssen; ein Stipendienprogramm à la BAföG existiert in Peru nicht und wird, das kann man wohl prognostizieren, aufgrund der finanziellen Situation des Landes so schnell auch nicht eingeführt. Damit verzögert sich aber die Dauer eines Studiums zwangsläufig; die Leistungsnachweise können nicht oder zumindest nicht rechtzeitig erbracht werden. Die Folge wäre eine Exmatrikulierung. So wird diese Neuregelung der tatsächlichen Situation im Lande nicht gerecht $^{32}$.

In der neuen Verfassung wurde zudem eine Bestimmung wie Art. 39 ('79) nicht wiederholt, wonach im Staatshaushalt mindestens 20 Prozent dem Erziehungssektor vorzubehalten waren. Diese - unrealistische - Forderung ist auch nie erfüllt worden ${ }^{33}$.

30 Enrique Obando Arbulú, Seguridad, Defensa Nacional y Fuerzas Armadas en la Constitución de 1993, in: Análisis Internacional 4 (Oktober-Dezember) Lima 1993, S. 67.

31 Die Angehörigen der Mittel- und Oberschicht schicken im allgemeinen ihre Kinder auf die Privatuniversitäten, die sich durch hohe Studiengebühren finanzieren müssen.

32 Diese Einschätzung wurde dem Verfasser im Gespräch mit Hochschullehrem in Peru wiederholt unterbreitet.

33

Rubio/Bernales, Constitución, S. 156. 


\subsection{Staat und Nation}

Die Republik von Peru, so der offizielle Name, versteht sich nach Art. 43 als demokratisch, sozial, unabhängig und souverän. Alle Staatsgewalt, so Art. 45, geht vom Volke aus. Niemand, keine Organisation, seien es die Streitkräfte, die Polizei oder ein anderer Sektor der Bevölkerung, dürfe sich diese Macht anmaßen. Dies bedeute Meuterei oder Rebellion. Besonders erwähnt wird dabei das Gebot der Gewaltenteilung. Ausdrücklich formuliert Art. 46 dann noch das Widerstandsrecht und berechtigt jeden Bürger zum Ungehorsam gegenüber jeglichen Anordnungen eines Usurpators. Dies sind alles Formulierungen, wie sie auch schon in den Art. $79 \mathrm{ff}$. ('79) standen. Traditionell basieren die demokratischen Verfassungen auf derartigen Prinzipien, Peru stellt insofern keine Ausnahme dar. Konkrete Ableitungen dürften jedoch auch hier schwierig sein.

Neben den üblichen Staatszielen wie Verteidigung der nationalen Souveränität, vollständige Garantie der Menschenrechte, Förderung des allgemeinen Wohls etc. wird in Art. 44 dem Staat noch die Verpflichtung zur Förderung speziell der lateinamerikanischen Integration auferlegt, ähnlich wie zuvor schon in Art. 100 ('79). Art. 54, wie schon Art. 98 ('79), weist ausdrücklich darauf hin, daß der peruanische Staat sein Territorium bis auf 200 Seemeilen auf das Meer hinaus ausgedehnt hat ${ }^{34}$. Dies entspricht der Politik der Pazifikanrainerstaaten aus der Zeit direkt nach dem 2. Weltkrieg. In einer Deklaration im Anhang zur neuen Verfassung hatte die Verfassunggebende Versammlung dann noch Peru, zur südlichen Hemisphäre gehörig, als Land mit historischen Ansprüchen auf die Antarktis erklärt, u.a. mit dem Recht auf eine, wie es heißt, vernünftige und ausgewogene Ausbeutung der dortigen Bodenschätze, ohne dabei die in dem Antarktisvertrag festgelegten Bedingungen außer Acht zu lassen.

Internationale Verträge müssen, nach Art. 56 enumerativ festgelegt, vom Kongreß gebilligt werden, wenn sie die folgenden vier Themenbereiche betreffen: 1. Menschenrechte, 2. staatliche Souveränität, 3. nationale Verteidigung und 4. finanzielle Verpflichtungen des Staates. Auch die Kündigung von Staatsverträgen dieser Art bedarf der vorherigen Billigung des Kongresses, Art. 57.

\subsection{Wirtschaftssystem}

Wer die Rechtsprechung des Bundesverfassungsgerichts zur wirtschaftspolitischen Neutralität des Grundgesetzes vor Augen hat, wird die Eindeutigkeit, mit der die neue peruanische Verfassung ein bestimmtes Wirtschaftssystem vorschreibt, recht ungewöhnlich finden. Art. 58 betont ausdrücklich die freie Privatinitiative. Sie entfalte sich in einem 
System der sozialen Marktwirtschaft. Unter diesem Regime leite der Staat die Entwicklung des Landes, hauptsächlich auf den Gebieten der Arbeitsplatzbeschaffung, des Gesundheits- und Bildungswesens, der Sicherheit, der öffentlichen Dienstleistungen und der Infrastruktur. Dabei stellt Art. 63 ausdrücklich die nationalen und die ausländischen Investitionen gleich, schließt aber für den Fall, daß ein ausländischer Staat protektionistische Maßnahmen ergreife, eine entsprechende Reaktion des peruanischen Staates nicht aus.

Bezüglich des Privateigentums legt Art. 71 fest, daß die Ausländer den Peruanern gleichgestellt sind; lediglich in einem Grenzstreifen von $50 \mathrm{~km}$ Breite ist Ausländern jegliches Eigentum an Land, Bodenschätzen, Wasser, Energie usw. verwehrt, auch in indirekter Form durch Beteiligung an einheimischen Gesellschaften, will man nicht die entschädigungslose Enteignung riskieren; dies entspricht Art. 126 ('79).

Für landwirtschaftlich nutzbare Flächen gibt es noch eine Besonderheit. Sie können, falls sie nicht bestellt werden, an den Staat zum Zweck ihres Verkaufs (und damit einer entsprechenden Nutzung) fallen; Einzelheiten bedürfen einer gesetzlichen Regelung, Art. 88. In Art. 157 ('79) gab es schon eine ähnliche Regelung; damals galt jedoch, daß derartige Flächen ausdrücklich an Bauern ohne Land abgegeben werden sollten. In diesem Zusammenhang sind dann im Rahmen der Landreform in den 70er Jahren Bestimmungen eingeführt worden, die die unmittelbare Bearbeitung des Ackers in den Vordergrund stellten; die - zivilrechtliche - Eigentümerposition hatte dann nur untergeordnete oder überhaupt keine Bedeutung. Ob derartige Regelungen jedoch unter der neuen Verfassung beibehalten werden, muß man abwarten; zumindest hat sich in der letzten Zeit eine Einstellung verfestigt, die das Eigentum als solches in der Vordergrund rückt.

Eine völlig neue Bestimmung ist Art. 64, wonach der freie Besitz und die Verfügungsgewalt über ausländische Währung garantiert wird. Gemeinhin finden sich (und befanden sich auch bis dato) derart spezielle Bestimmungen nicht in Verfassungen, da sie die Währungspolitik einer Regierung in unangemessener Weise beschränken könnten, ohne daß ihnen die in einer Verfassung angelegte grundsätzliche Bedeutung zukäme. Man glaubt wohl, nur durch eine verfassungsrechtliche Festlegung einer bestimmten Politik das Vertrauen der Bevölkerung wiedergewinnen zu können, welches aufgrund der sehr wechselvollen Währungspolitik der früheren Regierungen verloren gegangen ist.

Die Kritik an diesen wirtschaftspolitischen Vorstellungen beschränkt sich ganz allgemein darauf, daß damit, im Gegensatz zur früheren Verfassung, nunmehr dem Staat eventuell notwendige Interventionen in das Wirtschaftsgeschehen versagt seien, z.B. in Krisensituationen $^{35}$. Selbst wenn dieser Abschnitt in der neuen Verfassung nicht so umfangreich 
ist wie in der vorherigen, so bieten die Art. $58 \mathrm{ff}$. doch zahlreiche Möglichkeiten des staatlichen Eingriffs; vielleicht sogar mehr als früher, da die Formulierungen derart vage gestaltet sind, daß sie einen größeren Raum für extensive Interpretationen lassen ${ }^{36}$.

\subsection{Staatsorgane}

\subsubsection{Parlament}

Das Legislativorgan ist nach Art. 90 ein Ein-Kammer-Kongreß, eine wichtige Neuerung. Nach der Verfassung von 1979 bestand der auf fünf Jahre, parallel zur Amtsperiode des Staatspräsidenten gewählte Kongreß immer aus zwei Kammern, dem Senat und dem Abgeordnetenhaus. Die 60 Senatoren wurden in den Regionen gewählt. Regionen waren Verwaltungseinheiten und daher nicht vergleichbar mit den Bundesstaaten in den USA oder der Bundesrepublik, eher mit den französischen Departements. Die 180 Deputierten des Abgeordnetenhauses wurden nach dem Verhältniswahlrecht bestimmt. Dabei handelte es sich nicht um die Form des klassischen Zwei-Kammer-Systems, wobei die eine Kammer wegen ihrer landesweiten Legitimation sich vorwiegend übergreifenden nationalen Interessen verpflichtet fühlte, während die andere Kammer aufgrund ihrer regionalen Legitimation eher die Interessen der Provinzen vertreten sollte. Dieses System hatte in dieser Form in Peru nie funktioniert, da die dafür erforderliche Dezentralisierung und Regionalisierung in den früheren Verfassungen allenfalls auf dem Papier stand. Die Regionen hatten zu keiner Zeit funktionsfähige Organe mit eigenständiger Legitimation; sie waren vielmehr immer sowohl in administrativer wie insbesondere auch in finanzieller Hinsicht von der Zentralregierung in Lima unmittelbar abhängig ${ }^{37}$. So blockierten sich die beiden Kammern immer häufiger gegenseitig. Da sie nicht unterschiedlichen Interessen verpflichtet waren, konkurrierten sie gerade im Hinblick auf dieselbe Wählerklientel.

Schon bei der Verfassung von 1933 ist dies kritisiert worden, da der Senat gemeinhin nur ein Werkzeug der Oligarchie, d.h. der Großgrundbesitzer sowie der Industrie ${ }^{38}$ oder, schärfer noch, die Nachahmung einer nicht-existenten Aristokratie in Peru sei ${ }^{39}$. Diese grundsätzliche Kritik wurde auch gegen die Beibehaltung des Zwei-Kammer-Systems in der Verfassung von 1979 erhoben $^{40}$. Warum jetzt allerdings in der Einführung eines Ein-

Gabriel Ortiz de Zevallos M., La Constitución desde una perspectiva económica, in: Apuntes 33, Lima 1993, S. 13 ff.

37

Bürgermeister waren von der Bevölkerung direkt gewählt worden; aber die Gemeinden erhielten direkte Zuweisungen aus dem Staatshaushalt.

38

A. Ruiz Eldrigde, zit. bei Rubio/ Bernales, Constitución, S. 334.

39

40

César Delgado-Guembes, La Reforma del Parlamento, in: CAJ, No. 8, S. 44 [zit: C. DelgadoGuembes, CAJ No. 8].

Rubio/Bernales, Constitución, S. 335. 
Kammer-Parlaments ein Verstoß gegen die - vermeintlich positive - Verfassungstradition

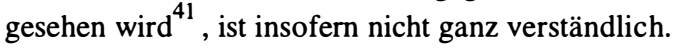

Als Präsident Fujimori bei seinem "autogolpe" im April 1992 auf die tatsächlich wohl mangelnde Effizienz dieses Zwei-Kammer-Systems hinwies, konnte er demnach in der Bevölkerung einer breiten Zustimmung gewiß $\operatorname{sein}^{42}$. Der ehemalige Zwei-KammerKongre $ß$ hatte in der Tat seiner Aufgabe als Kontrollorgan des Präsidenten und der Regierung nicht gerecht werden können. Die "Eifersüchteleien" zwischen den beiden Kammern führten weiterhin auch zu einer allenfalls begrenzten Effektivität, wenn nicht gar zu einer Verlangsamung bei der Gesetzgebung ${ }^{43}$, so daß zunehmend der Präsident mit Hilfe von Gesetzesdekreten regieren konnte.

Die Zahl der Kongreßabgeordneten ist auf 120 halbiert worden. Jetzt entfällt ein Deputierter auf rund 180.000 Einwohner. Damit liegt Peru am Ende der südamerikanischen Staaten, die von 45.000 (Bolivien) und bis 147.000 (Ekuador) Einwohner pro Abgeordneten reicht. Eine Ausnahme bildet Brasilien mit einem Verhältnis von $845.000: 1^{44}$. Die Wahlperiode beträgt fünf Jahre. Das Wahlsystem ist verfassungsseitig nicht festgelegt; es bleibt einem einfachen Gesetz überlassen. Sichergestellt sein muß nach Art. 176 allerdings, daß das Wahlsystem u.a. den authentischen Wählerwillen widerspiegelt. Eine Mehrheitswahl dürfte damit ausgeschlossen sein; vielmehr wird man es bei der schon jetzt üblichen Verhältniswahl belassen ${ }^{45}$. Da eine Sperrklausel fehlt, ist die Zahl der im Parlament vertretenen Parteien immer relativ groß gewesen. In der zuletzt gewählten Verfassunggebenden Versammlung waren insgesamt 10 Parteien vertreten, obwohl, wie eingangs erwähnt, die klassischen Parteien wie APRA und AP an dem Wahlgang nicht teilgenommen hatten. Und von diesen erwähnten Parteien entsandte eine Gruppierung, obwohl sie nur unter 2 Prozent der Stimmen erreicht hatte, immerhin noch ein Mitglied in die Konstituante. Die Mitgliederstärke der anderen Parteien, außer der der Regierungspartei mit 44 Abgeordneten, bewegte sich zwischen 2 und 8 Deputierten. Angesichts dessen ist eine Diskussion zur Einführung des Mehrheitswahlrechts zumindest unwahrscheinlich.

CAJ, Del golpe..., S. $248 \mathrm{ff}$.

Marcial Rubio, Gobernabilidad Democrática en el Perú, Análisis Internacional 3 (Juli-September) Lima, 1993, S. 68.

Rubio/Bernales, Constitución, S. 334.

44

Zusammenstellung bei José Luis Sardón, La reforma del parlamento, in: Apuntes 28, Lima 1991, S. 63.

45

Einige Überlegungen zur Änderung des Wahlrechts, auch mit Bezug auf das bundesdeutsche System, bei José Luis Sardón, El sistema de gobierno en la nueva constitución, in: Apuntes 33, Lima 1993, S. 9. 
Abgeordneter kann nur werden, wer Peruaner von Geburt ist, das 25. Lebensjahr vollendet hat und das allgemeine Wahlrecht besitzt. Präsidentschaftskandidaten dürfen sich nicht gleichzeitig als Listenkandidaten für den Kongreß aufstellen lassen, während dies den Bewerbern für die Vizepräsidentschaft gestattet ist, Art. 90. Der Hintergrund für diese zunächst einmal unverständliche Regelung dürfte sein, daß damit eine "Inflation" von Präsidentschaftskandidaten vermieden werden soll.

Regierungsmitglieder sowie Inhaber höchster Staatsämter, wie sie in Art. 91 aufgezählt werden (z.B. Verfassungsrichter oder der Präsident der Zentralbank), müssen ihre Ämter sechs Monate vor der Wahl niederlegen, wenn sie für den Kongreß kandidieren wollen. So soll wohl verhindert werden, daß die Kandidaten ihr Amt für den Wahlkampf mißbrauchen. Das gleiche gilt auch für im aktiven Dienst stehende Angehörige der Streitkräfte und der Nationalpolizei.

Die Tätigkeit eines Abgeordneten gilt nach Art. 92 als Vollzeitbeschäftigung, so daß, zumindest für die Stunden der Parlamentssitzungen, die Ausübung eines Berufs oder Amtes verboten ist. Das Abgeordnetenmandat selbst ist unvereinbar mit einem öffentlichen Amt, außer dem eines Ministers oder eines Kommissionsmitglieds auf internationaler Ebene. Gleiches gilt für den Abgeordneten, der Funktionen in privaten Unternehmen ausübt, die wiederum direkt oder indirekt mit dem Staat zu tun haben.

Art. 93 betont, daß die Abgeordneten das ganze Volk repräsentieren und in keiner Form einem imperativen Mandat unterworfen sind und garantiert ihnen Immunität und Indemnität. Eine Besonderheit stellt Art. 95 dar, wonach ein Abgeordneter nicht von dieser Funktion zurücktreten kann; interne disziplinarische Maßnahmen seitens des Kongresses dürfen deshalb 120 Tage innerhalb einer Legislaturperiode nicht überschreiten. Diese auch schon in Art. 178 ('79) bestehende Regelung war bereits kritisiert worden, da so ein einmal gewählter Abgeordneter nunmehr im Parlament agieren könne, auch wenn er aus der Partei ausgeschlossen sei ${ }^{46}$. Dementsprechend war schon die Aufhebung des Postulats des nicht-imperativen Mandats gefordert worden ${ }^{47}$. Auch eine andere Form der Abberufung aus einem parlamentarischen Mandat ist nicht möglich. Insoweit dürften Art. 2 Nr. 17 und Art. 31 nicht einschlägig sein, wonach das Volk nach bestimmten, vom Gesetz festzulegenden Formen staatliche Autoritäten abberufen kann, z.B. Friedensrichter (siehe weiter unten 2.5.4.).

Der Kongreß kann Untersuchungsausschüsse einrichten, für die die allgemeinen strafprozessualen Regeln gelten, Art. 97. Dabei kann auch die Aufhebung des Bank- und des Steuergeheimnisses verlangt werden. Nur die Privatsphäre bleibt ausgespart.

Rubio/Bernales, Constitución, S. 345.

C. Delgado-Guembes, CAJ No. 8, S. 45. 
Der Kongreß wählt aus seinen Mitgliedern für die sitzungsfreie Zeit eine Ständige Kommission, entsprechend dem Stärkeverhältnis jeder parlamentarischen Gruppe. Die Ständige Kommission darf laut Art. 101 nicht mehr als 25 Prozent der Gesamtzahl aller Kongreßmitglieder, also höchstens 30 Abgeordnete umfassen. Da voraussichtlich die Direktwahl von (parteiunabhängigen) Abgeordneten grundsätzlich nicht möglich sein wird, erübrigen sich entsprechende Regelungen für deren Repräsentanz. Die Möglichkeit, daß ein Deputierter fraktionslos wird, berücksichtigt die Verfassung nicht.

Die Aufgaben der Ständigen Kommission sind in Art. 101 enumerativ aufgeführt. Zum einen gehört dazu die Beteiligung an der Nominierung einiger der höchsten Staatsämter; zum anderen kann diese Kommission in gewissem Umfang legislative Kompetenzen des Kongresses übernehmen, allerdings nicht auf verfassungsrechtlichem Gebiet, bei der Annahme internationaler Verträge sowie bei der Verabschiedung von Organgesetzen und beim Haushaltsrecht. Im übrigen hat die Ständige Kommission keinerlei Befugnisse im Rahmen des Ausnahmezustands; hier ist allenfalls der Kongreß gefordert (siehe unten 2.5.3.4.)

Im Unterschied zu der Verfassung von 1979 erhält die Ständige Kommission jetzt einen eigenständigen Kompetenzbereich, so daß sie auch als "verkappter Senat" bezeichnet wird $^{48}$. Für diese Auffassung spricht u.a., daß vormals die Ständige Kommission nur aus 5 (von 60) Senatoren und 10 (von 180) Abgeordneten bestand, zuzüglich die beiden Kammerpräsidenten, also insgesamt 17 Mitglieder, während es heutzutage ja bis zu 30 Abgeordnete sein können. Auch wenn 1979 nicht so ganz klar war, welches denn nun die Aufgaben dieser Ständigen Kommission sein sollten ${ }^{49}$, so bestand doch Einigkeit dahingehend, daß diese Kommission lediglich einige Kompetenzen des Parlaments in dessen sitzungsfreier Zeit ausüben sollte ${ }^{50}$.

\subsubsection{Gesetzgebung}

Dem Kongreß obliegt die Gesetzgebung, die Verabschiedung des Haushalts, die Annahme internationaler Verträge, die Ausübung des Rechts der Amnestie und anderes mehr (Art. 102). Das Begnadigungsrecht steht jedoch nach Art. 118 Nr. 21 nur dem Staatsoberhaupt zu. Gesetze dürfen nicht zurückwirken, außer auf straf rechtlichem Gebiet zugunsten des Beschuldigten, Art. 103.

Enrique Bernales Ballesteros: La Comisión Permanente en la Constitución de 1993, in: Comisión Andina de Juristas: La Constitución de 1993, Lima 1994, S. 161.

Eine der peruanischen Verfassungstradition fremde Institution, die zuvor nur in der Verfassung von 1860 Eingang gefunden hatte, dann aber bereits durch eine Verfassungsänderung im Jahre 1874 wieder abgeschafft wurde.

Rubio/Bernales, Constitución, S. 335. 
Sehr weit gespannt ist der Kreis derer, die ein Gesetzesinitiativrecht haben, Art. 107. Zunächst einmal sind es der Staatspräsident und die Kongreßabgeordneten. Sodann steht dieses Recht auch hinsichtlich der sie betreffenden Rechtsmaterien den anderen Staatsgewalten $\mathrm{zu}$, den autonomen öffentlichen Institutionen, den Kommunen sowie den Berufsverbänden. So unterbreitet beispielsweise die Justiz durch den Obersten Gerichtshof eigenständig der Regierung ihren Haushaltsplan und vertritt ihn dann auch vor dem Kongreß, Art. 145. Ebenso besteht die Möglichkeit einer Volksinitiative; die Bedingungen dafür müssen in einem besonderen Gesetz niedergelegt werden.

Vom Parlament verabschiedete Gesetze werden dem Staatspräsidenten zugeleitet zur Ausfertigung binnen 15 Tagen, Art. 108. Sollte der Präsident die Ausfertigung nicht vornehmen, so übernimmt diese Aufgabe der Präsident des Kongresses oder, falls es in dessen Zuständigkeit gefallen ist, der der Ständigen Kommission. Sollte der Staatspräsident gegen das Gesetz oder Teile davon Bedenken hegen, so muß er diese innerhalb der erwähnten 15-Tage-Frist dem Kongreß mitteilen. Der Kongreß kann die Bedenken mit der Mehrheit seiner gesetzlichen Mitgliederzahl zurückweisen; dann obliegt dem Kongreßpräsidenten die Ausfertigung. Offenbar soll dem Staatspräsidenten die Peinlichkeit erspart bleiben, ein von ihm abgelehntes Gesetz ausfertigen zu müssen.

Im übrigen kann der Kongreß nach Art. 104 auf die Exekutive auch das Recht delegieren, Gesetzesdekrete zu erlassen. Dabei muß aber die Gesetzesmaterie spezifiziert und der Zeitraum für diese Delegation festgelegt werden. Nicht übertragbar sind allerdings die Bereiche, für die auch die Ständige Kommission nicht zuständig ist. Für Gesetzesdekrete gelten dann die gleichen formellen Voraussetzungen wie für normale Gesetze. Eine ähnliche Regelung enthielt schon die Verfassung von 1979. In einer Untersuchung für den Zeitraum Mitte 1980 bis Mitte $1989^{51}$ wurde festgestellt, da $ß$ nur rund zwei Drittel aller Gesetze vom Parlament verabschiedet wurden; alle übrigen waren Gesetzesdekrete. Bezieht man in diese Aufstellung auch noch die Notstandsdekrete mit ein, die vom Präsidenten aufgrund Art. 211 Nr. 20 ('79) erlassen werden konnten, so war der Kongreß nur bei weniger als der Hälfte aller Normen direkt beteiligt ${ }^{52}$. Dabei darf nicht übersehen werden, daß die beiden Präsidenten Belaúnde und García jeweils über eine ausreichende Mehrheit im Parlament verfügten, im Gegensatz zu Alberto Fujimori. Eine - mögliche Kontrolle durch das Parlament war also praktisch unterblieben ${ }^{53}$.

Auf einem Sektor hat es jedoch eine Neuerung gegeben, deren Auswirkungen noch nicht sichtbar sind. Früher war es dem Präsidenten möglich, qua Notstandsdekret direkt die

\footnotetext{
51 die Amtsperiode des Präsidenten Belaúnde (1980-1985) sowie vier Fünftel der Präsidentschaft von Alan García.

52

53

F. Eguiguren, Los retos..., S. 164.

CAJ, Del golpe..., S. 244.
} 
Steuergesetze zu ändern bzw. neue Steuern einzuführen oder auch Steuerarten wieder abzuschaffen. Dies geschah über eine sehr extensive Auslegung des Art. 211 Nr. 20 ('79), wonach dem Staatsoberhaupt die Möglichkeit eingeräumt worden war, auf dem Gebiet von Wirtschaft und Finanzen eben diese Notstandsdekrete zu erlassen ${ }^{54}$. Obwohl die neue Verfassung in Art. 118 Nr. 19 inhaltlich eine ähnliche Form gewählt hat, ist jedoch zuvor in Art. 74 ausdrücklich bestimmt worden, daß auf steuerrechtlichem Gebiet keine Notstandsdekrete erlassen werden dürften, was zumindest für die Wirtschaft von Interesse sein dürfte aufgrund der dadurch gestiegenen Rechtssicherheit, auch wenn eventuell die für notwendig erachtetet Stabilitätspolitik somit geschwächt werden könnte ${ }^{55}$. Zumindest werde so der (in der Vergangenheit häufig zu beobachtende) Mißbrauch des Notstandsrechts erheblich beschnitten ${ }^{56}$.

Insgesamt betrachtet reduziert sich die Kritik an diesem Teil der neuen Verfassung darauf, daß man die schon in der früheren Verfassung als ungenau bezeichneten Bestimmungen, die der Exekutive eine zu starke Position einräumten ${ }^{57}$, nicht genügend zugunsten einer effektiveren Gewaltenteilung geändert habe ${ }^{58}$.

\subsubsection{Regierung}

\subsubsection{Präsident}

Der Präsident der Republik, so definiert es Art. 110, ist Staatschef und personifiziert die Nation. Um Präsident zu werden, muß man Peruaner von Geburt an sein, bei Anmeldung der Kandidatur das 35. Lebensjahr vollendet haben sowie das allgemeine Wahlrecht besitzen.

Der Präsident und seine beiden Vizepräsidenten werden direkt gewählt, Art. 111. Dabei siegt, wer mehr als die Hälfte der abgegebenen Stimmen erhalten hat. Ungültige Stimmen und Stimmenthaltungen werden dabei nicht mitgezählt ${ }^{59}$. Wenn keiner der Kandidaten die absolute Mehrheit erhält, so findet innerhalb von 30 Tagen nach Veröffentlichung des

Zwischen 1980 und 1992 wurden insgesamt 2005 außerordentliche Dekrete erlassen, 1338 allein in der Amtszeit von Alan García (1985-1990).

Gabriel Ortiz de Zevallos M., La Constitución desde una perspectiva económica, in: Apuntes 33, Lima 1993, S. 17.

José Luis Sardón, El sistema de gobiemo en la nueva constitución, in: Apuntes 33, Lima 1993, S. 9.

Francisco José Eguiguren Praeli: La legislación delegada y los decretos de urgencia en la Constitución peruana de 1993, in: Comisión Andina de Juristas: La Constitución de 1993, Lima 1994, S. 177. CAJ, Del golpe..., S. 245.

59

Damit wurde ein alter Streit beigelegt; dazu: Francisco Eguiguren Praeli, La elección presidencial en la constitución de 1979: alcances y controversias en el sistema electoral vigente, in: Lecturas sobre temas constitucionales 4, Lima 1990, S. $77 \mathrm{ff}$. 
of fiziellen Wahlergebnisses eine Stichwahl zwischen den beiden bestplazierten Bewerbern statt. Diese Bestimmung entspricht Art. 203 ('79). Dadurch soll eine möglichst breite Legitimation erreicht werden.

Das Wahlverfahren ist aber nicht unumstritten. Keine politische Kraft könne nach siegreichem Urnengang von sich beanspruchen, deshalb die Mehrheit der Peruaner zu repräsentieren. Aus politischer Sicht sei die bei der Stichwahl erreichte Mehrheit eine "falsche Mehrheit"; ausreichend sollte es deshalb sein, daß beispielsweise 33 oder 40 Prozent der Wählerstimmen für die Wahl zum Präsidenten genügten ${ }^{60}$. Bei der Debatte um die Verfassung von 1979 war zudem noch der Verdacht geäußert worden, so könne am ehesten die wahrscheinliche relative Mehrheit eines APRA-Kandidaten durch ein Bündnis aller anderen Parteien im zweiten Wahlgang übertroffen werden ${ }^{61}$. Diese Ansicht übersieht allerdings, daß eine Stichwahl zumindest die Möglichkeit der Koalitionsbildung bietet, so da $ß$ der künftige Präsident mit einer ausreichenden Mehrheit im Parlament regieren kann. Zugegebenermaßen ist dies bis 1992 Theorie geblieben, denn bei der ersten Wahl nach der Militärdiktatur im Jahr 1980 bestand eine Ausnahmeregelung, wonach der Gewinner mindestens 36 Prozent der gültigen Stimmen auf sich vereinigen mußte, was Fernando Belaúnde auch gelang. Bei der Wahl von 1985 erhielt der APRA-Kandidat Alan García nur knapp unter 50 Prozent der Stimmen, woraufhin der Zweitplazierte, Alfonso Barrantes von der Vereinigten Linken, auf die Stichwahl verzichtete, zumal die APRA die Mehrheit der Sitze im Parlament gewonnen hatte. Bei dem Urnengang von 1990 reduzierte sich die Stichwahl auf eine - zumindest in der Öffentlichkeit so hochstilisierte Auseinandersetzung zwischen dem Repräsentanten der Großbourgeoisie, Mario Vargas Llosa, und dem Vertreter der Unterprivilegierten, Alberto Fujimori. Trotzdem könnte sich diese Möglichkeit in Zukunft als hilfreich erweisen, so daß eine Stichwahl durchaus zur zumindest formalen - Mehrheitsbildung auf den verschiedenen politischen Ebenen beitragen kann.

Nach Art. 112 beträgt die Amtszeit eines Präsidenten fünf Jahre, unmittelbare Wiederwahl ist einmal möglich ${ }^{62}$. Wenn man so will, kann nach dem Zwischenspiel eines anderen Präsidenten der Ex-Präsident erneut kandidieren, wiederum für zwei Amtsperioden. Dies ist eine sehr umstrittene Neuerung gegenüber der Verfassung von 1979, die eine unmittelbare Wiederwahl nicht zuließ. Befürchtet wird dabei, die Möglichkeit einer unmittelbaren Wiederwahl verstärke angesichts der geringen demokratischen Tradition des Landes die Neigung zu den in Lateinamerika bekannten autoritären Regimeformen.

60

Francisco Eguiguren Praeli, Reforma constitucional y poder ejecutivo, in: CAJ No. 8, S. 36 [zit: F. Eguiguren, CAJ No. 8].

61

Enrique Chirinos Soto, zitiert bei Rubio/Bernales, Constitución, S. 384.

62

In Lateinamerika (außer seit neuestem in Argentinien) bisher generell unüblich; in Ekuador wird derzeit eine ähnliche Neuregelung geplant. 
Im übrigen stelle diese neue Verfassungsnorm eine nachträgliche Legitimierung von Fujimoris Staatsstreich dar ${ }^{63}$. Andererseits wird, durchaus auch von Gegnern der neuen Verfassung, eingeräumt, daß der Ausschluß einer Wiederwahl zu dem "absurden" Ergebnis führen würde, daß ein vom Volk gewünschter Präsident an der Fortführung seiner Politik aus formalen Gründen gehindert werde; zu überlegen sei nur, ob nicht die Amtszeit von fünf auf vier Jahre verkürzt werden sollte, einer besseren Kontrolle wegen ${ }^{64}$. Im übrigen könne davon ausgegangen werden, daß ein Präsident, der in seiner ersten Amtszeit verantwortungsvoll (und nicht korrupt) gearbeitet habe, wohl diese Linie in der anschließenden Periode beizubehalten verspreche ${ }^{65}$.

Die Präsidentschaft endet nach Art. 113 durch Tod oder vom Kongreß akzeptierten Rücktritt, weiterhin dann, wenn der Kongreß moralische oder physische Amtsunfähigkeit des Präsidenten festgestellt hat (wohl sehr unwahrscheinlich). Ebensolches gilt, wenn der Präsident ohne die notwendige Erlaubnis des Kongresses das Land verläßt oder innerhalb der gesetzten Frist nicht zurückkehrt (keine Exilpräsidentschaft). Weiterhin ist ein Amtsenthebungsverfahren möglich, wenn der Präsident gegen bestimmte, in Art. 117 aufgeführte Verfassungsgrundsätze verstößt. Dazu zählen Vaterlandsverrat, Verhinderung der Wahlen auf allen Ebenen, unzulässige Auflösung des Kongresses bzw. Beeinträchtigung von dessen Funktionsfähigkeit etc. Alles in allem dürfte wohl ein Amtsenthebungsverfahren, obwohl die verschiedenen Gründe im einzelnen aufgeführt werden, kaum realisiert werden $^{66}$, trotz des brasilianischen Gegenbeispiels.

Die Kompetenzen des Präsidenten sind im einzelnen in Art. 118 aufgeführt. Er ist, wie bei einer Präsidialverfassung üblich, oberster staatlicher Repräsentant und bestimmt auch die generelle politische Linie der Regierung. So muß der Präsident jährlich zu Beginn der Sitzungsperiode des Kongresses eine Regierungserklärung abgeben. Außer zu Beginn der Legislaturperiode bedarf diese Regierungserklärung der vorherigen Zustimmung des Kabinetts. Des weiteren ernennt er nach Genehmigung durch das Kabinett u.a. die Botschafter und muß davon den Kongreß nur in Kenntnis setzen. Nach der früheren Verfassung hatte dabei z.B. der Senat ein Mitbestimmungsrecht. Wichtig ist, daß der Präsident dem System der Nationalen Sicherheit vorsteht und, zumindest formell, dessen materielle und personelle Struktur festlegt (dazu weiter unten 2.5.6). Des weiteren hat der Präsident notstandsrechtliche Kompetenzen, die aber nachträglich vom Kongreß modifiziert oder aufgehoben werden können.

Carlos Gandolfo, El régimen parlamentario y la estabilidad democrática, in: Apuntes 29, Lima 1991, S. 21. ist jedoch auch nach seinem Staatsstreich nicht in Erwägung gezogen worden. 


\subsubsection{Ministerrat}

Die Leitung und Ausführung der öffentlichen Verwaltung obliegen dem Ministerrat (Kabinett), wobei jeder Minister nach dem Ressortprinzip für seinen Amtsbereich verantwortlich ist, Art. 119. Handlungen des Präsidenten, denen die ministerielle Gegenzeichnung fehlt, sind nichtig, Art. 120.

Dem Ministerrat sitzt der Ministerpräsident vor, der wiederum vom Staatspräsidenten ernannt und entlassen wird. Die Minister werden auf Vorschlag und im Einvernehmen mit dem Ministerpräsidenten vom Staatspräsidenten ernannt und entlassen, Art. 121, 122. Die Mitglieder des Kabinetts müssen peruanische Bürger von Geburt und mindestens 25 Jahre alt sein; auch Angehörige der Streitkräfte und der Nationalpolizei im aktiven Dienst können dem Kabinett angehören, Art. 124.

Der Ministerpräsident, der - im Gegensatz zur früheren Verfassung - kein Ressort leiten muß, hat zunächst eine koordinierende Funktion, Art. 123. Er ist, nächst dem Staatspräsidenten, der qua Verfassung autorisierte Sprecher der Regierung. (Den Vizepräsidenten fällt diese Stellung nicht zu; sie haben im übrigen keine besonderen Aufgaben, außer der Vertretung des Präsidenten.) Im übrigen muß der Ministerpräsident die Gesetzes- und Notstandsdekrete gegenzeichnen.

Das Kabinett hat, neben der Beratung von Angelegenheiten im öffentlichen Interesse, die Gesetzentwürfe des Präsidenten vor Einbringung in den Kongreß sowie u.a. die Gesetzesund Notstandsdekrete zu billigen, Art. 125. Daß die Mitglieder des Ministerrats keine anderweitigen privaten und kommerziellen Interessen wahrnehmen dürfen, versteht sich fast von selbst, Art. 126. Nach Art. 128 sind die Minister gemeinschaftlich verantwortlich für Verfassungs- und Gesetzesverstöße, die dem Präsidenten vorgeworfen oder die im Ministerrat beschlossen wurden, es sei denn, sie treten unmittelbar zurück.

Ein substantieller Unterschied zu der Verfassung von 1979 ist nicht erkennbar; vielmehr ist es immer noch der Präsident, der die Politik bestimmt. Der Ministerpräsident und das Kabinett sind immer noch unmittelbar vom Präsidenten abhängig; auch nur ansatzweise ist eine Eigenständigkeit des Ministerrats gegenüber dem Staatschef nicht ersichtlich.

\subsubsection{Verhältnis Exekutive - Legislative}

Innerhalb von 30 Tagen nach Amtsübernahme unterbreitet der Ministerpräsident dem Kongreß sein Regierungsprogramm. Dabei stellt er die Vertrauensfrage, Art. 130. Die Konsequenzen bei einer Abstimmungsniederlage werden nicht genannt. Man wird deshalb Art. 134 heranziehen müssen. Danach kann der Präsident im Wiederholungsfall das Parlament auflösen und Neuwahlen ausschreiben ${ }^{67}$.

67

José Luis Sardón, El sistema de gobiemo en la nueva constitución, in: Apuntes 33, Lima 1993, S. 8. 
Der Kongreß hat zunächst ein Interpellationsrecht gegenüber dem Ministerrat insgesamt wie auch gegenüber einzelnen Ministern, Art. 131. Diese Interpellation muß von mindestens 15 Prozent der gesetzlichen Mitgliederzahl der Abgeordneten initiiert werden; zur Annahme bedarf es dann der Zustimmung eines Drittels der Deputierten. Diese Quoren entsprechen Art. 225 ('79). Die Abstimmung muß unmittelbar in der nächsten Parlamentssitzung erfolgen. Die Befragung des oder der Minister ist dann zwischen dem 3. und dem 10. Tag nach der Annahme durchzuführen ${ }^{68}$.

Der Kongreß kann dem Ministerrat oder einzelnen Minister sodann das Mißtrauen aussprechen bzw. das Vertrauen verweigern, Art. 132. Für die Vertrauensfrage bedarf es einer Initiative seitens der Minister. Ein Mißtrauensantrag auf der anderen Seite muß von mindestens 25 Prozent der gesetzlichen Mitgliederzahl der Kongreßabgeordneten gestellt werden. Debatte und Abstimmung über den Antrag erfolgen innerhalb des 4. und 10. Tages nach seiner Einbringung. Zu seiner Annahme bedarf es der Mehrheit aller Kongreßabgeordneten. Im Falle des Unterliegens müssen der oder die betroffenen Minister dann zurücktreten. Der Staatspräsident akzeptiert, so heißt es lapidar, diese Demission innerhalb von 72 Stunden.

Der Ministerpräsident kann jederzeit im Namen des gesamten Kabinetts die Vertrauensfrage stellen, Art. 133. Wird das Vertrauen verweigert bzw. ist ein Mißtrauensantrag erfolgreich, tritt das gesamte Kabinett geschlossen zurück.

Der Präsident hat das Recht, den Kongreß aufzulösen und Neuwahlen auszuschreiben, wenn dieser zweimal den Kabinetten das Mißtrauen ausgesprochen bzw. das Vertrauen verweigert hat, Art. 134. Dies gilt aber nur für den Ministerrat insgesamt, nicht für einzelne Mitglieder. Eine ähnliche Bestimmung kannte schon die frühere Verfassung, allerdings mußte damals der Dissens zwischen Regierung und Mehrheit im Abgeordnetenhaus dreimal in dieser Weise dokumentiert werden, Art. 227 ('79). Eine Auflösung des Senats war damals grundsätzlich nicht möglich, Art. 230 ('79), zumal der Senat in diesen Konflikt zu keinem Zeitpunkt eingebunden war.

Das Auflösungsdekret des Präsidenten enthält gleichzeitig den Termin zur Neuwahl, die innerhalb von vier Monaten erfolgen muß; dafür darf das existierende Wahlsystem nicht geändert werden. Allerdings ist die Auflösung des Kongresses im letzten Jahr der Legislaturperiode unzulässig. Das gleiche Verbot gilt auch während des Ausnahmezustandes. Nach der Auflösung des alten bis zum Zusammentritt des neuen Kongresses fungiert

68 Diese und andere exakte Zeitbestimmungen in der Verfassung deuten auf ein nur unterentwickeltes Vertrauen auf demokratische Gepflogenheiten und Geschäftsordnungen bzw. deren Respektierung hin. 
weiterhin die Ständige Kommission. Ein Selbstauflösungsrecht des Kongresses gibt es nicht.

Die Legislaturperiode des neugewählten Kongresses endet jedoch mit Ablauf der Amtszeit des Staatspräsidenten, d.h. es besteht eine direkte Verknüpfung zwischen dem Tätigkeitszeitraum von Exekutiv- und Legislativorgan. Sollte es innerhalb der 4-Monats-Frist nicht zu Neuwahlen kommen, tritt der bereits aufgelöste Kongreß wieder mit allen seinen Rechten zusammen; der Ministerrat muß dann zurücktreten, und keines seiner Mitglieder darf in der verbleibenden Amtsperiode des Präsidenten erneut zum Minister berufen werden, Art. 136. Fraglich ist, ob diese Bestimmungen ausreichen, um einen möglichen Konflikt zwischen dem Präsidenten und einer oppositionellen Mehrheit im Kongreß zu lösen.

Auch die frühere Verfassung kannte, wie bereits dargelegt, die Form der Auflösung der Abgeordnetenkammer und der Neuwahl nach dreimaliger Ablehnung des gesamten Kabinetts. Diese Frequenz auf zweimal reduziert zu haben kann schwerlich als qualitativer Fortschritt angesehen werden. Insofern unterstellt auch die neue Verfassung offenbar, da $\beta$ ein Präsident über eine ausreichende Unterstützung im Kongreß verfügt. Bei den früheren Präsidenten Belaúnde und García war das der Fall gewesen. Erst das jetzige Staatsoberhaupt stand nach seiner Wahl 1990 einem von der Opposition mehrheitlich beherrschten Parlament gegenüber mit dem Ergebnis des "autogolpe" im April 1992.

Fraglich ist zudem, inwieweit auch bei den Abgeordneten ein Interesse an einer Parlamentsauflösung besteht, laufen sie doch Gefahr, ihr Mandat und die diesem innewohnenden Privilegien zu verlieren. Es ist eher zu vermuten, daß die Deputierten durch Mißtrauensanträge nur gegenüber einzelnen Kabinettsmitgliedern (und nicht gegenüber dem gesamten Kabinett) die Regierung in Schwierigkeiten zu bringen versuchen. Permanente Krisen zwischen Parlament und Regierung könnten also weiterhin die Folge sein. Tatsächlich ist zwischen 1980 und 1992 auch nur ein Minister, und zwar ein Landwirtschaftsminister des Präsidenten Fujimori, vom Parlament zum Rücktritt gezwungen worden. In den vorherigen Regierungen gab es keine erzwungene Ministerdemission. Irgendwelche substantiellen Konsequenzen aus dieser politischen Konstellation sind aber in der neuen Verfassung nicht erkennbar; sie bietet also keine neuen Lösungsansätze für eventuelle Konflikte.

Eine andere Einschätzung könnte sich aus der Stellung des Ministerrats ergeben. Daß es nunmehr möglich ist, einen Ministerpräsidenten zu bestellen, ohne daß dieser zugleich ein Ressort übernehmen muß, er also mehr ist als ein primus inter pares (zumal ihm zusätzlich noch eine nach außen hin gerichtete Verantwortlichkeit beigemessen wird, Art. 130), dürfte für sich genommen aber noch keine Verbesserung sein. Wichtiger erscheint, daß 
der Ministerpräsident zu Beginn seiner Amtszeit die Vertrauensfrage stellen muß $\beta^{69}$. Das bedeutet, er, oder besser gesagt, der Staatspräsident muß gleich zu Beginn einer Legislaturperiode eine ausreichende Mehrheit durch Koalitionsbildung oder ähnliche Absprachen herstellen. Scheitert dies, so kann der Präsident wiederum nur mit einer Minderheitsregierung arbeiten und muß von Fall zu Fall Mehrheiten im Kongreß suchen. Sollte ihm dies nicht gelingen, so kann er eventuell, falls es das nationale Interesse (wie immer dies auch definiert werden mag) erfordert, mit Notstandsmaßnahmen arbeiten, was ihm nach Art. 118 Nr. 19 auch im Rahmen der Wirtschafts- und Finanzpolitik (mit Ausnahme des Steuerrechts, s.o.) möglich ist. Und gerade bei diesen Sektoren sind die Auseinandersetzungen - nicht nur in Peru - selbstverständlich am ernstesten. Der Kongreß kann diese Maßnahmen allenfalls im nachhinein modifizieren oder aufheben.

Die politische Praxis der nächsten Jahre muß erweisen, ob diese neue Regelung tatsächlich die erwünschte größere Stabilität bewirken kann. Die Möglichkeit des Präsidenten, mit Notstandsmaßnahmen zu regieren, ist zumindest nicht entscheidend eingeschränkt worden, so daß die neue Verfassung auch in diesem Punkt letztlich keinen substantiellen Fortschritt gebracht hat.

\subsubsection{Ausnahmezustand}

Bei Staatskrisen kann der Präsident für das ganze Land (oder auch nur in Teilen) den Ausnahmezustand ausrufen. Dabei wird unterschieden zwischen dem estado de emergencia, Art. 137 Nr. 1, und dem estado de sitio, Art. 137 Nr. 2. Der estado de emergencia gilt für schwere innenpolitische Krisen und Katastrophen. Dabei können bestimmte Grundrechte eingeschränkt oder vollständig außer Kraft gesetzt werden, beispielsweise die persönlichen Freiheitsrechte, die Unverletzlichkeit der Wohnung, die Versammlungsfreiheit und die Freizügigkeit.. Das entsprechende Dekret wird vom Präsidenten im Einvernehmen mit dem Kabinett erlassen; der Kongreß muß von diesen Maßnahmen nur in Kenntnis gesetzt werden. Die Dauer des estado de emergencia beträgt höchstens 60 Tage; Verlängerungen sind möglich, ohne daß der Kongreß sein Plazet gegeben muß.

Der estado de sitio kann ausgerufen werden bei Krieg oder Bürgerkrieg. Es reicht allerdings schon, wenn eine derartige Gefahr droht. Dann sind alle Grundrechte quasi automatisch außer Kraft gesetzt; gegebenenfalls müssen die weiter bestehenden Rechte ausdrücklich benannt werden. Die Zeitdauer beträgt höchstens 45 Tage; eine Verlängerung bedarf der Genehmigung durch den Kongreß.

\subsubsection{Rechtsprechung}

Art. 139 enthält, zumindest formell, die üblichen Garantien im Hinblick auf ein rechtsstaatliches Verfahren durch eine unabhängige Justiz. Der unabhängige Nationale Richter- 
rat nimmt die Auswahl und Ernennung der Richter und Staatsanwälte vor, Art. 150. Nach Art. 154 Nr. 2 müssen Richter und Staatsanwälte auf allen Ebenen nach sieben Jahren erneut in ihren Ämtern bestätigt werden. Im Falle der Nichtbestätigung müssen sie aus dem Richterdienst bzw. aus der Anklagebehörde ausscheiden. Daß dieses Damoklesschwert der möglichen Nichtbestätigung schwerlich der richterlichen Unabhängigkeit dienlich ist ${ }^{70}$, bedarf keiner weiteren Begründung.

Der Nationale Richterrat setzt sich zusammen aus mindestens sieben, in geheimer Abstimmung gewählten Mitgliedern: 1 vom gemeinsamen Senat des Obersten Gerichtshofs, 1 von der Gruppe der Obersten Ankläger, 1 von den Mitgliedern der Anwaltskammern, 2 von den Mitgliedern der übrigen berufsständischen Kammern, 1 von den Rektoren der staatlichen Universitäten, 1 von den Rektoren der privaten Universitäten. Die Zahl der Mitglieder kann um 2 erweitert werden; sie werden von dem Rat selbst ausgewählt aus Listen, die ihm repräsentative Vereinigungen der Unternehmer und der Arbeitnehmer vorlegen. Um in den Nationalen Richterrat gewählt werden zu können, muß man Peruaner von Geburt sein, die bürgerlichen Rechte besitzen und das 45. Lebensjahr vollendet haben.

Nach der Verfassung von 1979 fiel dem Nationalen Richterrat nur die Aufgabe zu, dem Staatspräsidenten die Richter zur Ernennung vorzuschlagen; die Berufung der Richter am Obersten Gerichtshof bedurften zusätzlich der Bestätigung durch den Senat, Art. 245 ('79). Durch die Neuregelung erhofft man sich, den unmittelbaren Einfluß von Exekutive und Legislative auf die Zusammensetzung der Judikative zurückdrängen zu können ${ }^{71}$.

Art. 153 verbietet es Richtern und Staatsanwälten zudem, sich politisch zu betätigen, Gewerkschaften zu bilden und in den Streik zu treten.

Die - zumindest zahlenmäßig - gewichtigste Institution innerhalb der Justiz bilden die Friedensrichter, nach deutschem Recht am ehesten den Schiedsleuten vergleichbar. Als Instanz von geringerer Bedeutung auf zivil- und strafrechtlichem Gebiet ${ }^{72}$ stellen sie ca. 90 Prozent der peruanischen Richterschaft ${ }^{73}$ und werden durch Volkswahl bestimmt; ebensolches kann gesetzlich für die ("normalen") Richter der 1. Instanz festgelegt werden, Art. 152. Vormals wurden die Friedensrichter von den örtlich zuständigen Oberlandesgerichten (Cortes Superiores) ausgewählt.

CAJ: Del golpe..., S. 259.

Javier de Belaunde L. de R.: Elección popular de jueces, in: Comisión Andina de Juristas: La Constitución de 1993, Lima 1994, S. 204.

72

Zusammenfassend Fermín G. Chunga Lamon ja, La justicia de paz en el Perú, Lima 1979.

73

Juan Monroy Gálvez, El poder judicial a 10 años de vigencia de la constitución de 1979, in: Lecturas sobre temas constitucionales 4, Lima 1990, S. 68. 
Nunmehr soll nach Art. 139 Nr. 17 durch Gesetz die Beteiligung der Bevölkerung bei der Wahl und der Abberufung von Richtern geregelt werden. Gerade die Möglichkeit der Entfernung aus dem Amt kollidiert jedoch mit Art. 146, der den Richtern die bekannte richterliche Unabhängigkeit einschließlich einer angemessenen Besoldung garantiert. Kritisch gilt auch, was schon weiter oben im Hinblick auf die Bestätigung bzw. Nichtbestätigung von Richtern gesagt wurde.

Durch die unmittelbare Partizipation der Bevölkerung könnten nunmehr (partei-) politische oder ökonomische Interessen in unzulässiger Weise diese Mitbestimmung beeinflus$\operatorname{sen}^{74}$. Eingedenk des im allgemeinen schlechten Ansehens der peruanischen Justiz in der Bevölkerung (Stichwort: Korruption) vermögen solche Bedenken jedoch nicht zu überzeugen, könnte sich doch durch die Volkswahl gerade der Richter auf der untersten Ebene zumindest ansatzweise eine Rechtsprechung entwickeln, die auch einer kritischen Öffentlichkeit standhält.

Die Richterwahl und -abberufung wird vielleicht verständlich vor dem Hintergrund des Art. 149. Danach können die Autoritäten der Comunidades Campesinas y Nativas, also der bäuerlichen und indigenen Gemeinschaften (vornehmlich im abgeschiedenen Andenhochland sowie im Dschungelgebiet), zusammen mit den Rondas Campesinas, den zunächst gegen Viehdiebe und später gegen Terroristen gerichteten Bauernwehren, justizielle Funktionen in ihrem Sprengel ausüben, auf der Grundlage von Gewohnheitsrecht, soweit die Grundrechte aus der Verfassung nicht verletzt werden. Durch Gesetz müssen die Formen der Koordination speziell zwischen den Friedensrichtern (Juzgados de Paz) und den übrigen Gerichtsinstanzen festgelegt werden.

Aus dem Rahmen fällt die besondere Stellung der Militärjustiz. Zu deren Aufgaben gehört(e) die Aburteilung von Angehörigen der Streitkräfte wegen dienstlicher Vergehen, sowohl nach altem, Art. 282 ('79), wie auch nach neuem Recht, Art. 173. Der Art. 282 ('79) wurde zunächst als ein Fortschritt gegenüber der herkömmlichen Praxis bezeichnet. Damit sollte die Möglichkeit einer Erweiterung des Tätigkeitsbereichs der Militärtribunale auf die die Streitkräfte nur mittelbar betreffenden Gebiete ausgeschlossen werden. Gerade zu Zeiten der Militärregierungen in Peru zwischen 1968 und 1980 hatte man mit dieser Art Justiz grundsätzlich schlechte Erfahrungen gemacht ${ }^{75}$. In den letzten Jahren hatte sich für Rubio/Bernales jedoch ein ganz anderes Problem ergeben. Seit Mitte der 80er Jahre sei es im Rahmen der Bekämpfung des Terrorismus, besonders des "Sendero Luminoso", wiederholt zu Übergriffen der Sicherheitskräfte speziell gegenüber der ländli-

Javier de Belaunde L. de R.: Elección popular de jueces, in: Comisión Andina de Juristas: La Constitución de 1993, Lima 1994, S. 214.

75 Marcial Rubio / Enrique Bernales: Peru: constitución y sociedad política, 1. Aufl. Lima 1981, S. 440 . 
chen Bevölkerung in den Anden gekommen. Wurde nun z.B. ein Soldat zwecks Beobachtung von "subversiven" Elementen an einen bestimmten Ort geschickt und hat er zur "Abschreckung" dabei einen Menschen umgebracht, so läge nach den für Zivilisten geltenden strafrechtlichen Regeln ein mit hoher Gefängnisstrafe belegtes Tötungsdelikt vor; für den Soldaten käme aber eventuell ein minder schwer bestrafter Exzeß bei der Befehlserfüllung in Betracht ${ }^{76}$. Rubio/Bernales führen einige Beispiele an, wonach der Oberste Gerichtshof bei der Zuweisung von derartigen Fällen an eine zivile oder eine militärische Strafkammer eine stringente Entscheidungsfindung habe vermissen lassen ${ }^{77}$.

Hier ist also die notwendige Präzisierung des Art. 282 ('79) in dem Art. 173 unterblieben. Über die Gründe läßt sich nur spekulieren; vermutlich wollte die Verfassunggebende Versammlung einen - dann unausweichlichen - Konflikt mit den Streitkräften vermeiden. Sodann konnten Militärgerichte nach der alten Verfassung nur bei Landesverrat im Kriegsfall Todesurteile auch gegenüber Zivilpersonen verhängen, Art. 282 i.V.m. Art. 235 ('79). Jetzt können Prozesse gegen Terroristen, also Nicht-Militärs, vor Militärgerichten stattfinden. Lediglich deren Todesurteile werden gegebenenfalls einer Revision durch den Obersten Gerichtshof unterworfen (Art. 141 i.V.m. Art. 173), nicht jedoch beispielsweise eine Verurteilung zu lebenslanger Haft.

Im Gegensatz zu Art. 238 ('79), wonach der jährliche Haushalt zwei Prozent für die Justiz bereitstellen mußte, ist eine derartige Regelung in die neue Verfassung nicht aufgenommen worden. Die (alte) Verfassungsvorschrift ist (wie auch die entsprechende Norm im Erziehungsbereich) ohnehin nie eingehalten worden ${ }^{78}$.

\subsubsection{Bürgerbeauftragter}

Der Bürgerbeauftragte (und seine Behörde) soll hauptsächlich über Rechte der Bürger gegenüber staatlichen Ämtern wachen, ohne daß jedoch konkret ausgeführt wird, wie er dies tun soll. Das erinnert an den "Ombudssmann" skandinavischer Prägung. Er wird vom Kongreß mit einer Zwei-Drittel-Mehrheit auf fünf Jahre gewählt und unterliegt nicht einem imperativen Mandat. Seine Unabhängigkeit gleicht der der Obersten Richter. Er muß mindestens 35 Jahre alt und Jurist sein. Mindestens einmal jährlich erstattet er dem Kongreß Bericht; im übrigen hat er auch ein Initiativrecht im Gesetzgebungsverfahren. Der Bürgerbeauftragte ist nur bedingt mit dem Ministerio Público in der Verfassung von 1979 (dort Art. 250) zu vergleichen; daher wird man die Funktion und Bedeutung dieser Institution erst nach dem Ablauf von ein paar Jahren herausarbeiten können.

Rubio/Bernales, Constitución, S. 441 f.

Rubio/Bernales, Constitución, S. 442 ff; s.a. amnesty international: Peru - Klima des Terrors, Bonn 1991, S. $117 \mathrm{ff}$.

78 Juan Monroy Gálvez, El poder judicial a 10 años de vigencia de la constitución de 1979, in: Lecturas sobre temas constitucionales 4, Lima 1990, S. 71. 


\subsubsection{Nationale Sicherheit und Verteidigung}

Die innere Sicherheit obliegt grundsätzlich der Nationalpolizei (zu deren Aufgabenbereich im übrigen auch die Grenzsicherung gehört). Während des Ausnahmezustands (Art. 137) können die Streitkräfte dazu herangezogen werden. Im übrigen haben Heer, Marine und Luftwaffe die Unabhängigkeit, Souveränität und territoriale Integrität des Landes zu wahren, Art. 163 ff. Sodann nehmen Streitkräfte und Polizei nach Art. 171 an der ökonomischen und sozialen Entwicklung des Landes teil (Arbeits- und Katastropheneinsätze?). Ausdrücklich wird klargestellt, daß die Sicherheitskräfte nicht eine sog. beratende Funktion haben, sondern sich der verfassungsmäßigen Ordnung unterordnen müssen, Art. $169^{79}$. Daß diese (und andere) Bestimmungen jedoch vor einem Staatsstreich schützen könnten, wird ernsthaft von niemandem angenommen. Denn immer noch versteht sich das Militär in Lateinamerika als "Hüter der Verfassung" und nahm sich in der Vergangenheit wiederholt unter diesem Motto das "Recht" zum Putsch heraus ${ }^{80}$.

Die Konstitutionalität der Armee wird wohl am ehesten erreicht werden können, wenn es dem Präsidenten gelingt, auf die Zusammensetzung der Führungsebene entscheidenden Einfluß auszuüben. Art. 172 schreibt vor, daß der Präsident die Beförderungen von Generälen und Admiralen der Streitkräfte sowie der Nationalpolizei vornimmt, entsprechend den Vorschlägen der jeweiligen Institution. Nach der ehemaligen Verfassung bedurfte die Beförderung von obersten Dienstgraden der Ratifikation durch den Senat.

Gem. Art. 167 ist der Präsident weiterhin Oberbefehlshaber (Jefe Supremo) der Armee sowie der Polizei, ohne daß dies präzisiert wird ${ }^{81}$. Jedoch wird in der Beförderung allein durch den Präsidenten eine Stärkung seiner Position gesehen ${ }^{82}$. Rubio meint nicht ganz zu Unrecht, es sei sinnvoll, dem Präsidenten die Möglichkeit einzuräumen, an die Spitze der Sicherheitskräfte das Personal zu berufen, welches sein Vertrauen besitzt ${ }^{83}$. Dahinter steht der seit der Unabhängigkeit des Landes nie entschiedene Streit um den Primat der Politik und die Frage, ob und inwieweit die Politik auch (vermeintlich) interne Angelegenheiten der Armee bestimmen dürfe. Immer noch wird (nicht nur in Peru) die Auffassung vertreten, das Militär sei eine unpolitische Organisation, allenthalben dazu berufen, ihre Interna selbständig zu regeln, bis hin zur Bestellung ihrer obersten Führungs-

79

80

Heinrich-W. Krumwiede, $\mathrm{Zu}$ den Überlebenschancen von Demokratie in Lateinamerika, in: Lateinamerika Jahrbuch 1993, Frankfurt/M 1993, S. 15.

81

82

83

CAJ, Del golpe..., S. 239.

CAJ, Del golpe..., S. 254.

Marcial Rubio, Las Fuerzas Armadas en la Nueva Constitución Peruana, in: E. Obando A., Fuerzas Armadas y Constitución, Lima 1993, S. 54. 
organe $^{84}$. Nur so könne deren Intervention, sprich: ein Staatsstreich, verhindert werden. Im Verlauf der vergangenen 170 Jahre hatte sich die Praxis entwickelt, daß die Streitkräfte nach dem Anciennitätsprinzip praktisch völlig autonom ihre Stäbe besetzten; dem jeweiligen Präsidenten oblag nur, diese Entscheidungen abzuzeichnen. Erst Präsident Fujimori hat mit dieser Praxis gebrochen, sehr zum Mißvergnügen der Generalität, die jedoch, wie sonst üblich, angesichts der ökonomischen und politischen Schwierigkeiten im Land nicht ernsthaft mit einem Putsch drohen konnte. Sollte diese Politik fortgesetzt werden, so könnte Peru zu einem Land werden, wo mittelf ristig der periodisch stattfindende Staatsstreich der Vergangenheit angehört.

\subsubsection{Wahlsystem}

In diesem Kapitel wird nicht, trotz der Überschrift, im einzelnen festgelegt, nach welchem System in Peru gewählt werden muß, z.B. durch ein Mehrheits- oder ein Verhältniswahlrecht. Einzig in Art. 176 werden allgemeine Voraussetzungen für eine demokratische Wahl als Ausdruck des Volkswillens benannt. Vielmehr beschränken sich die Art. $177 \mathrm{ff}$. darauf, die Struktur und Aufgaben des Jurado Nacional de Elecciones (JNE), also des Nationalen Wahlausschusses, festzulegen.

Ähnlich wie auch bei dem Nationalen Richterrat ist die Zusammensetzung ungewöhnlich, Art. 179. Der Ausschuß besteht aus fünf Mitgliedern im Alter zwischen 45 und 60 Jahren. Ein Mitglied, welches gleichzeitig den Vorsitz innehat, wird vom Obersten Gerichtshof aus dem Kreis seiner noch im Dienst befindlichen oder auch pensionierten Richter gewählt. Ein weiteres Mitglied wählen die obersten Ankläger aus den Reihen der aktiven oder pensionierten Staatsanwälte. Sodann wählt der Anwaltsverein von Lima aus der dortigen Anwaltschaft ein Mitglied. Die Dekane der Rechtsfakultäten sowohl der staatlichen wie der privaten Universitäten wählen jeweils ein Mitglied aus dem Kreis der ExDekane. Gem. Art. 180 handelt es sich dabei um eine Vollzeittätigkeit für eine Periode von vier Jahren; eine Wiederwahl der Mitglieder ist möglich. Die Arbeit muß entsprechend vergütet werden; eine andere Tätigkeit ist unzulässig, mit Ausnahme einer Dozentur.

Die Aufgaben des Wahlausschusses bestehen nach Art. 178 in der Vorbereitung und Durchführung einer Wahl sowie der Feststellung des Ergebnisses. Seine Entscheidungen - auch im Rahmen einer Wahlprüfung - sind abschließend. ${ }^{85}$ Selbstverständlich dürfen Kandidaten für die anstehende Wahl sowie hohe Parteifunktionäre (auch wenn sie derar-

Carlos Pellegrini, Diskussionsbeitrag, in: E. Obando A., Fuerzas Armadas y Constitución, Lima 1993, S. $86 \mathrm{f}$.

Domingo García Belaúnde, Sistema electoral y representación política en el Perú actual, in: Fundación Friedrich Ebert/ Instituto de Cooperación Iberoamericana, Sistemas electorales y representación política en Latinoamérica, Madrid 1986, S. 61. 
tige Funktionen in den letzten vier Jahren ausgeübt haben) nicht Mitglieder im Wahlausschuß werden, Art. 180.

In einem Fall hat die Verfassung jedoch vorgeschrieben, wie ein Wahlergebnis zu bewerten ist. In Art. 184 wurde festgelegt, daß eine Wahl dann für nichtig erklärt werden muß, wenn die Zahl der Stimmenthaltungen und der ungültigen Stimmen insgesamt mehr als 2/3 der abgegebenen Stimmen ausmacht; für Kommunalwahlen kann durch Gesetz ein anderes Verhältnis festgelegt werden. Diese Regelung entspricht Art. 290 ('79). Bedenklich sei, so Eguiguren, daß diese Norm in der Vergangenheit tatsächlich schon wiederholt angewendet werden mußte; ein Zeichen dafür, daß die Bevölkerung dem demokratischen Prozeß offenbar mit großer Skepsis gegenüberstehe ${ }^{86}$.

\subsection{Verfassungsrechtliche Garantien}

Die Verfassungsmäßigkeit von Gesetzen (und anderen Normen) sowie staatlichem Handeln gegenüber einzelnen Bürgern soll durch ein Verfassungsgericht (Tribunal Constitucional) garantiert werden, Art. $200 \mathrm{ff}$. Eine wichtige Neuerung ist hierbei, daß nun nicht nur die Gesetze und Gesetzesdekrete, sondern auch die Notstandsdekrete einer verfassungsgerichtlichen Überprüfung unterzogen werden können, was die Verfassung von 1979 nicht ermöglichte.

Trotz erster Überlegungen in diese Richtung in den 20er und 30er Jahren ${ }^{87}$ ist ein Verfassungsgericht (Tribunal de Garantías Constitucionales) erst durch die Verfassung von 1979 eingerichtet worden. Von den insgesamt neun Richtern wurden je drei vom Kongreß, von der Regierung und vom Obersten Gerichtshof ausgewählt; die Amtszeit betrug sechs Jahre, eine Wiederwahl war möglich. Dabei mußte alle zwei Jahre ein Drittel der Richter neu bestimmt werden, Art. 296, 297 ('79). In der Realität bedeutete dies, daß die Regierung und die sie tragende Parlamentsmehrheit zwischen 1980 und 1990 zwei Drittel der Richterstellen besetzen konnte.

Hier sieht die Verfassung wichtige Änderungen vor, Art. 201: Das Gericht besteht nunmehr aus sieben Mitgliedern, die auf fünf Jahre gewählt werden; eine unmittelbare Wiederwahl ist unzulässig. Die Wahl erfolgt durch den Kongreß mit der Zwei-Drittel-Mehrheit seiner gesetzlichen Mitglieder. Im übrigen müssen die Richter dieselben Qualifikationen erfüllen wie für die Wahl zum Obersten Gerichtshof. Zudem dürfen keine Richter und Staatsanwälte zu Verfassungsrichtern gewählt werden, die nicht ein Jahr zuvor ihre

Francisco J. Eguiguren Praeli, El Tribunal de Garantías Constitucionales: las limitaciones del modelo y las decepciones de la realidad, in: Comisión Andinas de Juristas, Lecturas sobre temas constitucionales No. 7, Lima 1991, S. 18 ff. [zit: F. Eguiguren, CAJ No. 7]. 
berufliche Tätigkeit aufgegeben haben. Damit soll verhindert werden, daß Richter, die direkt aus dem Justizdienst in das Verfassungsgericht überwechseln, eine zu große Zurückhaltung an den Tag legen könnten, wenn es darum geht, die Urteile ihrer ehemaligen Kollegen zu überprüfen ${ }^{88}$. Problematisch bleibt jedoch, und das galt auch zuvor für die Verfassung von 1979, die allzu kurze Amtsperiode sowie die - wenn auch jetzt nicht unmittelbare - Möglichkeit der Wiederwahl der Verfassungsrichter. Die Gefahr politischer oder anderer Pressionen bleibt bestehen ${ }^{89}$.

Laut Art. 203 sind zur Anrufung des Verfassungsgerichts wegen Verfassungswidrigkeit von Gesetzen und anderer Normen befugt: der Staatspräsident, der Oberste Ankläger, der Bürgerbeauftragte, ein Viertel der Kongreßmitglieder, eine Gruppe von mindestens 5.000 Bürgern (ausweislich ihrer Unterschriften), Regionalpräsidenten und Bürgermeister sowie die berufsständischen Kammern bei den sie betreffenden Materien. Interessant ist hierbei u.a., daß, im Gegensatz zur vorherigen Verfassung, der Oberste Gerichtshof nicht mehr zum Kreis der Berechtigten gehört. Weiterhin wurde die Zahl der klagebefugten Bürger von vormals 50.000 auf jetzt 5.000 gesenkt. Des weiteren können nunmehr auch die im Rahmen der geplanten Regionalisierung berufenen Autoritäten das Verfassungsgericht anrufen.

Die Urteile des Gerichts haben quasi legislative Kraft und gelten ab dem Tag nach der Veröffentlichung, Art. 204. Auch hier gibt es einen nicht unwichtigen Unterschied zur Verfassung von 1979, denn nach Art. 301 ('79) teilte das Gericht dem Parlament seine Entscheidung über die Verfassungswidrigkeit eines Gesetzes mit; die Legislative hatte dann die Möglichkeit, die Norm in einem förmlichen Verfahren abzuschaffen. Sollte das Parlament nicht tätig werden, so trat die Bestimmung nach Ablauf von 45 Tagen automatisch außer Kraft; das Gericht ordnete dabei die Veröffentlichung seiner Entscheidung im Staatsanzeiger an. Dies war und ist insofern von Bedeutung, als sowohl nach Art. 300 ('79) wie nach Art. 204 ein derartiges Urteil keinerlei Rückwirkung entfaltet, sondern erst mit der Veröffentlichung in Kraft tritt. Das konnte nach der vorherigen Verfassung bedeuten, daß innerhalb der 45-Tage-Frist trotz des verfassungswidrigen Gesetzes noch Maßnahmen ergriffen werden konnten, die danach nicht mehr möglich gewesen wären ${ }^{90}$. Diese Gefahr ist durch die Neuregelung ausgeschlossen worden.

Jorge Danós Ordóñez: Aspectos orgánicos del Tribunal Constitucional, in: Comisión Andina de Juristas: La Constitución de 1993, Lima 1994, S. 295.

90 Ordóñez, a.a.O.

F. Eguiguren, CAJ No. 7, S. 47. 
Auch wenn eine Bilanz der Tätigkeit des Verfassungsgerichts für den Zeitraum von 1983 bis 1992 nicht günstig ausfällt ${ }^{91}$, so sollte der Maßstab nicht schärfer sein als bei den übrigen Verfassungsorganen, deren Arbeit wohl auch nicht in allen Fällen positiv bewertet werden kann. Die Einrichtung eines Verfassungsgerichts war u.a. der Ausdruck eines tiefsitzenden Mißtrauens gegenüber der bis dato einerseits als politisch urteilenden sowie andererseits als korrupt geltenden Justiz, so daß durch eine Verfassungsreform nur eine Verbesserung erreicht werden kann ${ }^{92}$. Selbst wenn man die Auswahl der Verfassungsrichter nunmehr als zu politisch charakterisiert ${ }^{93}$, so war die bisher übliche Praxis auch nicht dazu angetan, sie beizubehalten. Wiederholt war die rechtzeitige Neuwahl eines Verfassungsrichters unterblieben, so daß das entsprechende Quorum innerhalb des Spruchkörpers bei den Entscheidungen nicht erreicht werden konnte ${ }^{94}$.

\subsection{Verfassungsänderung}

Verfassungsänderungen können nach Art. 206 initiiert werden durch den Staatspräsidenten (nach Genehmigung durch den Ministerrat), durch die Kongreßmitglieder sowie durch 0,3\% der Wahlbevölkerung (nach Art. 306 ('79): 50.000 Unterschriften) ${ }^{95}$. Zur Annahme der Verfassungsänderung bestehen zwei Möglichkeiten: a) der Kongreß genehmigt die Änderung mit der absoluten Mehrheit seiner gesetzlichen Mitgliederzahl, anschließend entscheidet eine Volksabstimmung (die Verfassung von 1979 hatte hier kein Referendum vorgesehen); b) der Kongreß stimmt in zwei aufeinanderfolgenden ordentlichen Sitzungsperioden jeweils mit einer Zwei-Drittel-Mehrheit der gesetzlichen Mitgliederzahl dafür. Nach Art. 306 ('79) bedurfte es einer absoluten Mehrheit in den beiden Kammern, und zwar in den beiden aufeinanderfolgenden ersten ordentlichen Sitzungsperioden ${ }^{96}$. Eine Parallelität zwischen den beiden Verfassungen existiert insofern, als daß der Staatspräsident kein Vetorecht hat wie bei den einfachen Gesetzen.

Javier Valle-Riestra, El fracaso de la constitución, in: CAJ No. 8, S. 20; F. Eguiguren, Los retos..., S. 60.

92

CAJ, Del golpe..., S. 222.

CAJ, Del golpe..., S. 262.

94 F. Eguiguren, CAJ No. 7, S. 49.

95

Bei dem Referendum 1993 ging man aus von einer Bevölkerung von rund 22.128.000 Einwohnern; wahlberechtigt waren 52,3\%, also rund 11.573 .00 Peruaner; danach bedürte es bei einem Volksbegehren zur Verfassungsänderung ca. 34.718 Unterschriften.

Die erste ordentliche Sitzungsperiode findet traditionsgemäß in dem Zeitraum vom 28.Juli bis zum 15. Dezember eines jeden Jahres statt. 
Eine "Ewigkeitsklausel" wie in Art. 79 III GG existiert in der neuen peruanischen Verfassung nicht. Auch die vorherigen Verfassungen schwiegen sich dazu aus, mit Ausnahme der Verfassung von 1933, wonach die Einführung der Möglichkeit einer Wiederwahl des Präsidenten ausdrücklich untersagt wurde, und der von 1839, die die Gewaltenteilung und das repräsentative Regierungssystem für unabänderlich erklärte.

\section{Schlußbetrachtung}

Eine abschließende Beurteilung dieser neuen peruanischen Verfassung muß differenziert ausfallen; zum einen hinsichtlich der Frage, ob dazu überhaupt die Wahl einer verfassunggebenden Versammlung notwendig war, und zum anderen, ob hier entscheidende Neuerungen eingeführt wurden, die diesen Auf wand nachträglich rechtfertigen.

$\mathrm{Zu}$ der ersten Frage kann verwiesen werden auf eine in Lateinamerika nicht unübliche Praxis, nämlich immer dann, wenn einschneidende Verfassungsänderungen geplant werden, nicht die übliche, von der Konstitution vorgezeichnete Form zu wählen, sondern gleich zur Einberufung einer besonderen verfassunggebenden Versammlung zu schreiten $^{97}$. Insofern stellt der peruanische Weg keine Besonderheit dar. Zudem stellte sich der Weg einer Verfassungsänderung als reichlich kompliziert und zeitauf wendig dar.

Die andere Frage ist sicherlich schwieriger zu beantworten, da naturgemäß einschlägige Erfahrungen mit dieser neuen Verfassung noch fehlen. Carlos Torres y Torres Lara hat ungefähr 180 Neuerungen gegenüber der Verfassung von 1979 gezählt; dabei habe es auf drei Gebieten fundamentale Änderungen gegeben: bei den Grundrechten, beim ökonomischen System sowie bei dem Verhältnis zwischen den verschiedenen Gewalten im Staat $^{98}$.

Tatsächlich wurden die partizipativen Rechte der Bevölkerung (zumindest theoretisch) erheblich erweitert. Auch wenn es in Zukunft noch der Umsetzung dieser verfassungsmäßigen Rechte bedarf, so ist zumindest ein Grundstein gelegt worden. Gerade bei der Wahl und der möglichen Abwahl der Friedensrichter (und der Richter der 1. Instanz) wird dies vermutlich am schwierigsten, gerade vor dem Hintergrund richterlicher Unabhängigkeit.

Der interpretatorische Versuch, in den Regelungen über das Wirtschaftsleben eine grundsätzliche Neubewertung der Aufgaben des Staates in einer tendenziell marktwirtschaftli- 
chen Ordnung zu sehen ${ }^{99}$, erscheint denn doch etwas vermessen. Dazu sind die im Gegensatz zur früheren Verfassung noch verschwommener formulierten Artikel kaum geeignet $^{100}$.

Kaum akzeptabel dürfte es sein, aus der Möglichkeit des Mißtrauensvotums des Kongresses gegenüber dem Kabinett bereits abzuleiten, hier seien erste Schritte hin zu einer parlamentarischen Regierungsform eingeleitet worden; auch für Peru bleibt es angesichts der starken Stellung des Staatschefs weiterhin bei einem überwiegend präsidentiellen System $^{101}$. Und das bedeutet, wie sonst in Lateinamerika auch, da $ß$ das Parlament nur unzureichend seiner Funktion als Kontrollorgan, geschweige denn als politischer Mitgestalter gerecht werden kann $^{102}$. Im übrigen verweist Nohlen auch zurecht darauf, daß in dem klassischen Land des Präsidentialismus, in den USA, dem Präsidenten mit dem Föderalismus ein starkes Gegengewicht gegenübersteht, das in den zentralistisch orientierten Staaten Lateinamerikas unbekannt ist ${ }^{103}$.

Die neue peruanische Verfassung enthält nicht das an sich notwendige System der checks and balances, wie es gerade in einer Präsidialverfassung unbedingt erforderlich ist, um so einen notwendigen Machtausgleich gewährleisten zu können. Es sind keine ausreichenden Ausgleichsmechanismen eingebaut; auch die neue Verfassung bietet keine allseits akzeptablen Wege der Konfliktlösung.

Einerseits kann das Parlament relativ einfach die Regierungsarbeit blockieren; die Möglichkeit des Präsidenten, nach Art. 137 mit Dekreten zu regieren, ist dann nur die unzulängliche Kompensation eines demokratischen Defizits. Andererseits kann der Kongreß allenfalls punktuell auf die Regierungsarbeit Einfluß nehmen. Sollte er einem Kabinett nicht das Vertrauen aussprechen, so drohen ihm im Wiederholungsfall Auflösung und Neuwahlen. Eine "cohabitación" nach französischem Vorbild ist nicht vorgesehen, kann aber, zumindest theoretisch, nicht ausgeschlossen werden. Dann würde allerdings die Regierungsgewalt auf das Parlament übergehen ${ }^{104}$. Dies ist jedoch eine recht spekulative Annahme. Generell gilt: die Stellung des eigentlich politisch Verantwortlichen, des Präsidenten, bleibt von dieser Auseinandersetzung unberührt. Allenfalls mittelfristig könnte eine derartige Kontroverse Auswirkungen zeitigen, nämlich bei einer eventuell anstehenden Wiederwahl des Staatsoberhaupts.

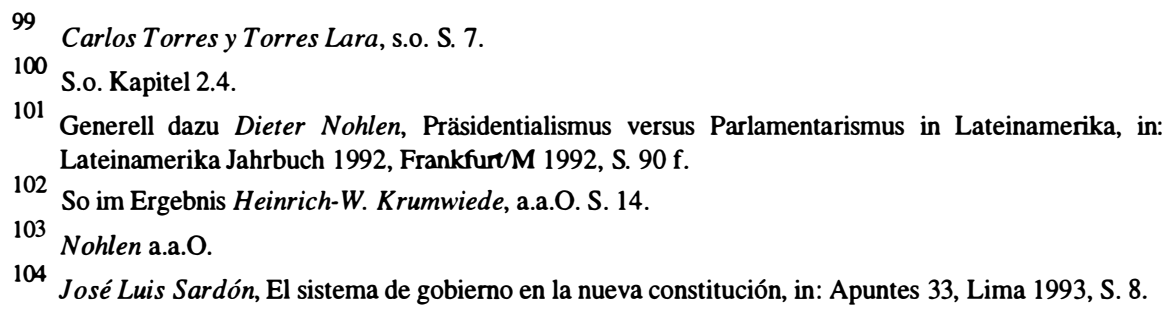


Zusammenfassend läßt sich sagen, daß diese neue peruanische Verfassung nicht notwendigerweise in der von dem Präsidenten Fujimori gewählten Form, also durch eine Staatsstreich, hätte initiiert werden müssen. Die Unterschiede zu der Verfassung von 1979 sind auch kaum so spektakulär, als daß dies nicht auch auf dem Weg einer Verfassungsänderung hätte erreicht werden können. Zugegebenermaßen wird man ein Urteil über diese neue Verfassung aber erst nach ein paar Jahren fällen können, wenn sie sich in der politischen Praxis bewährt hat (oder auch nicht). 


\title{
The Constitution of Peru of 1993
}

\author{
By Jürgen Saligmann
}

After his "coup d'état from above" in April 1992, Peru's president Alberto Fujimori came under intense international pressure. He therefore caused a constituent assembly to be elected which produced a new constitution ratified by referendum at the end of 1993. On the basis of the 1979 constitution, itself the result of a non-democratic period of government, certain modifications were added whose impact on the political system of Peru cannot yet be precisely assessed.

The provisions on basic rights have not been fundamentally changed apart from the introduction of capital punishment for terrorist acts. Changes are of a largely peripheral nature, mainly adapting the law to existing circumstances. The same cannot be said of the provisions on the organisation of government where the possibility to re-elect an incumbent president was introduced and the bicameral parliament replaced by a unicameral congress. The relationship of the presidency and congress has likewise been remoulded, but time will have to tell whether this has strengthened the position of parliament.

\section{The Jamaican Constitution in Litigation of the Covenant on Civil and Political Rights - Repercussions and Resonances}

\section{By Margaret DeMerieux}

Jamaica is an adherent to the Optional Protocol to the International Covenant on Civil and Political Rights, which permits persons within its jurisdiction to bring individual complaints before the U.N. Human Rights Committee.

Proceedings before the Committee engage the Constitution in two ways. The international law requirement that domestic remedies be exhausted before recourse is had to international proceedings, requires the Committee to examine the Jamaican Constitution to determine compliance with this rule. Domestic remedies arise under the Constitution. This leads to the second point of 'interrelation' between domestic law and the Constitution and Covenant. The Constitution contains a Bill of Rights, which has its roots in the same family of instruments on human rights (and in particular the Universal Declaration) as the Covenant. As a result, provisions of the Bill of Rights bear a striking resemblance to those of the Covenant and especially Article 14 thereof constituting the right to fair trial. The interpretation of that Article by the Committee has implications therefore, for the interpretation of the analogous Bill of Rights provision in the domestic law. This is highlighted in the decision of Morgan and Pratt v. A.G. (Jamaica) as discussed in this article. 